\title{
Road collisions avoidance using vehicular cyber-physical systems: a taxonomy and review
}

\author{
Faisal Riaz ${ }^{1}$ and Muaz A. Niazi ${ }^{2^{*}}$
}

*Correspondence:
muaz.niazi@gmail.com
${ }^{2}$ Department of Computer
Sciences-COMSATS,
Islamabad, Pakistan
Full list of author information
is available at the end of the
article

${ }^{*}$ Correspondence: muaz.niazi@gmail.com Sciences-COMSATS

Islamabad, Pakistan article

\begin{abstract}
Road traffic is known to have its own complex dynamics. One implication of complexity is that road traffic collisions have become an unwelcome but unavoidable part of human life. One of the major causes of collisions is the human factor. While car manufacturers have been focusing on developing feasible solutions for autonomous and semi-autonomous vehicles to replace or assist human drivers, the proposed solutions have been designed only for individual vehicles. The road traffic, however, is an interaction-oriented system including complex flows. Such a system requires a complex systems approach to solving this problem as it involves considering not only pedestrians, road environment, but also road traffic which can include multiple vehicles. Recent research has demonstrated that large-scale autonomous vehicular traffic can be better modeled using a collective approach as proposed in the form of vehicular cyber-physical systems (VCPS) such as given by Li et al. (IEEE Trans Parallel Distrib Syst 23(9):1775-1789, 2012) or Work et al. (Automotive cyber physical systems in the context of human mobility. In: National workshop on high-confidence automotive cyber-physical systems, Troy, MI, 2008). To the best of our knowledge, there is currently no comprehensive review of collision avoidance in the VCPS. In this paper, we present a comprehensive literature review of VCPS from the collision-avoidance perspective. The review includes a careful selection of articles from highly cited sources presented in the form of taxonomy. We also highlight open research problems in this domain.
\end{abstract}

Keywords: Agent based modelling, Complex system, Cyber physical system, Collisions, Emotions, Road traffic, Vehicular cyber physical system

\section{Background}

Road traffic dynamics are complex in nature. According to Zhao (2011), road traffic demonstrates various complex systems properties like non-uniformity, non-linearity, and adaptability, hence, it can be considered as complex in nature. Manley (2014) notices that individual driver behavior and unpredictable movement choices are the key reasons of complexity in road traffic. In another research study conducted by Doniec et al. (2008), it is noted that the interaction of heterogeneous road users like vehicles, pedestrians, and cyclists make the road traffic a complex phenomenon. These complex road traffic dynamics imply that it can be difficult to understand the exact dynamics of road traffic. Often times, such systems are analyzed from the individual perspective. This 
is equivalent to limited the view of the forest and examining only from the perspective of the trees.

Due to the complex nature of road traffic, collisions are an unavoidable part of human life. World Health Organization (WHO) notes annual road collisions as the cause of almost 1.2 million deaths globally (WHO 2007). Particularly important to note here is that among these deaths, the younger population is more highly affected as reported by Patton et al. (2009). This matter is even worse in the case of people from underdeveloped countries which have a higher death rate in road collisions primarily due to a lack of proper road infrastructure as noted by Fink (2014). Pakistan bureau of statistics (Gulzar et al. 2012) has reported an annual increasing death rate of 3100 people in road traffic. Examining this situations, it is clear that human factors appear to be the key reasons in making road collisions unavoidable.

Human drivers are one of the major reasons of road collisions. According to Rumschlag et al. (2015) human drivers are the major reason of accidents due to various careless activities such as talking on phone or texting. Chan and Singhal (2013) note that cognitive distraction has become one of the major reasons of road collisions. Making or listening to phone calls also make human drivers one of the major reasons of the road collisions as noted by Lansdown et al. (2015). The prime focus of auto industry has been on introducing different levels of autonomy in individual vehicles but not much on handling a large number of vehicles. The forward collision warning system (FCWS) was introduced in Volvo cars as autonomy level 1 (Bengler et al. 2014). It only detects the chances of collisions and alerts the drivers in advance. In 2011 (Schittenhelm 2013), autonomous braking system was introduced by Mercedes-Benz in the S-Class model as autonomy level 1 . Adaptive cruise control and lane-keeping functions were introduced in Tesla Model S vehicle as autonomy level 2 (Vogt 2016). Currently, Google a non-automaker company has tested its autonomous car, which meets autonomy level 4 (Lukic et al. 2008). However, these autonomous driving solutions for individual vehicles do not address all traffic related problems.

One possibility to provide comprehensive solutions to traffic-related problems is the vehicular cyber-physical system (VCPS). According to Reddy (2015), providing safer road environment is one of the goals of VCPS. According to Wolf (2014), vehicle control and operation is one of the classic CPS applications. According to Poovendran (2010), one of the basic function of CPS is the achievement of accident-free and efficient road transport. Autonomous vehicles (AVs), adaptive cruise control (ACC), lane departure warning, and early collision avoidance systems are the different types of VCPS. These VCPS are assisting humans without having the humans inspired design, which indicates an obvious cooperation gap between both current VCPS and drivers.

To the best of our knowledge, there is no comprehensive review of VCPS based collision avoidance in research literature. In this survey paper, we present a comprehensive review of VCPS based CAS. Further, open research problems have been discussed to indicate future research directions for the VCPS researchers.

Remaining paper is structured as follows. A comprehensive review regarding collision avoidance techniques is presented in "Collision avoidance using VCPS: a review" section. "Open research problems" section discusses open research problems. The paper is concluded in "Conclusions" section. 


\section{Collision avoidance using VCPS: a review}

In this section the comprehensive review of VCPS based road collision techniques have been presented.

\section{Road collisions taxonomy}

The road collisions taxonomy is shown in Fig. 1.

\section{A. Position based}

Position based collisions can be divided into two subtypes: rear end and lateral/lane departure.

a. Rear end

Definition According to Cabrera et al. (2012) rear-end collision is a transportation accident from where an agent assaults the back of another agent or vehicle.

The rear-end collision scenario is shown in Fig. 2. Rear-end collisions have a major role in deaths and injuries happened in the USA. According to Harb et al. (2007) rear end collisions alone contributed one-third of the 6 million stated crashes in the USA in 2003. Furthermore, in 2009 total 3.54 million rear-end crashes happened in the USA and caused 1.078 million injuries and 2100 fatalities as reported by Chen et al. (2015). Also, front and rear end collisions have a substantial contribution in automotive-related trauma and long term injuries than other types of road collisions as noted by Nishimura et al. (2015). According to Poplin et al. (2015), front-rear end collisions cause 9000 cases of severe abdominal injuries every year in the US only. From these statistics, it is very much obvious that how important is to tailor the efficient rear end collision avoidance solutions.

\section{b. Lateral/lane departure/blind spot}

Definition In lateral collision two vehicles traveling in parallel direction collides with each other side by side (Mon and Lin 2012).

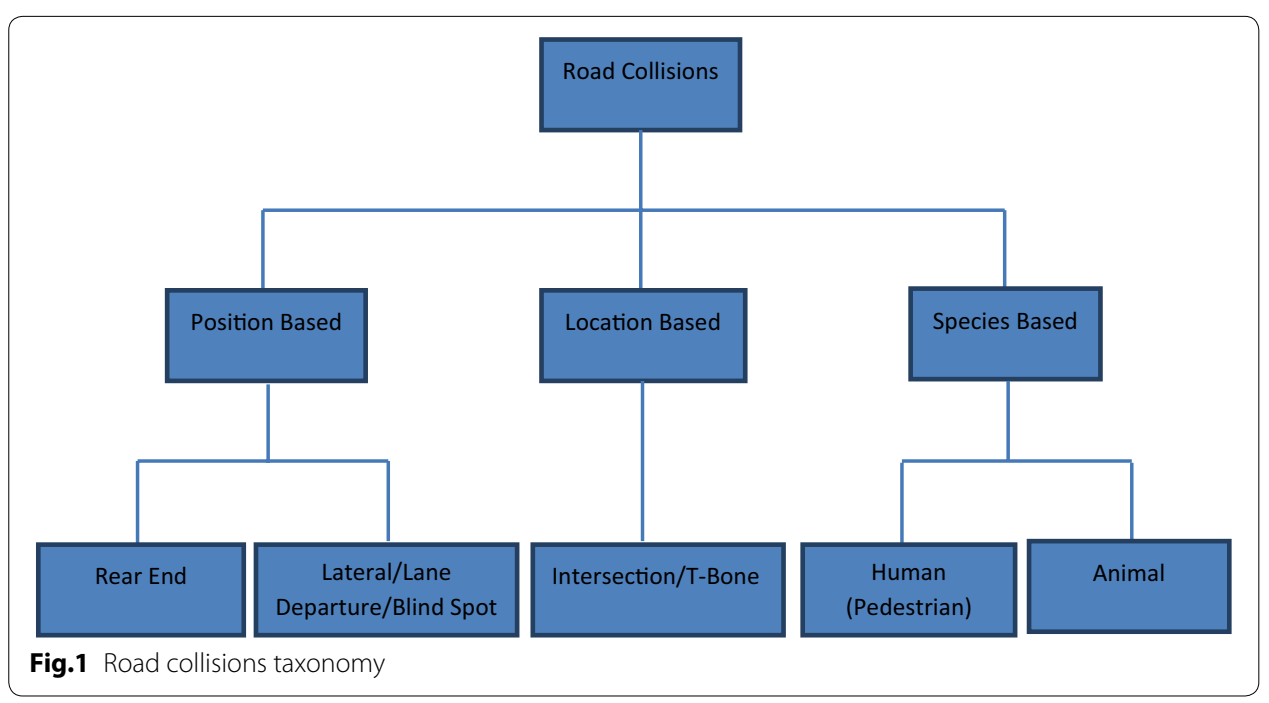



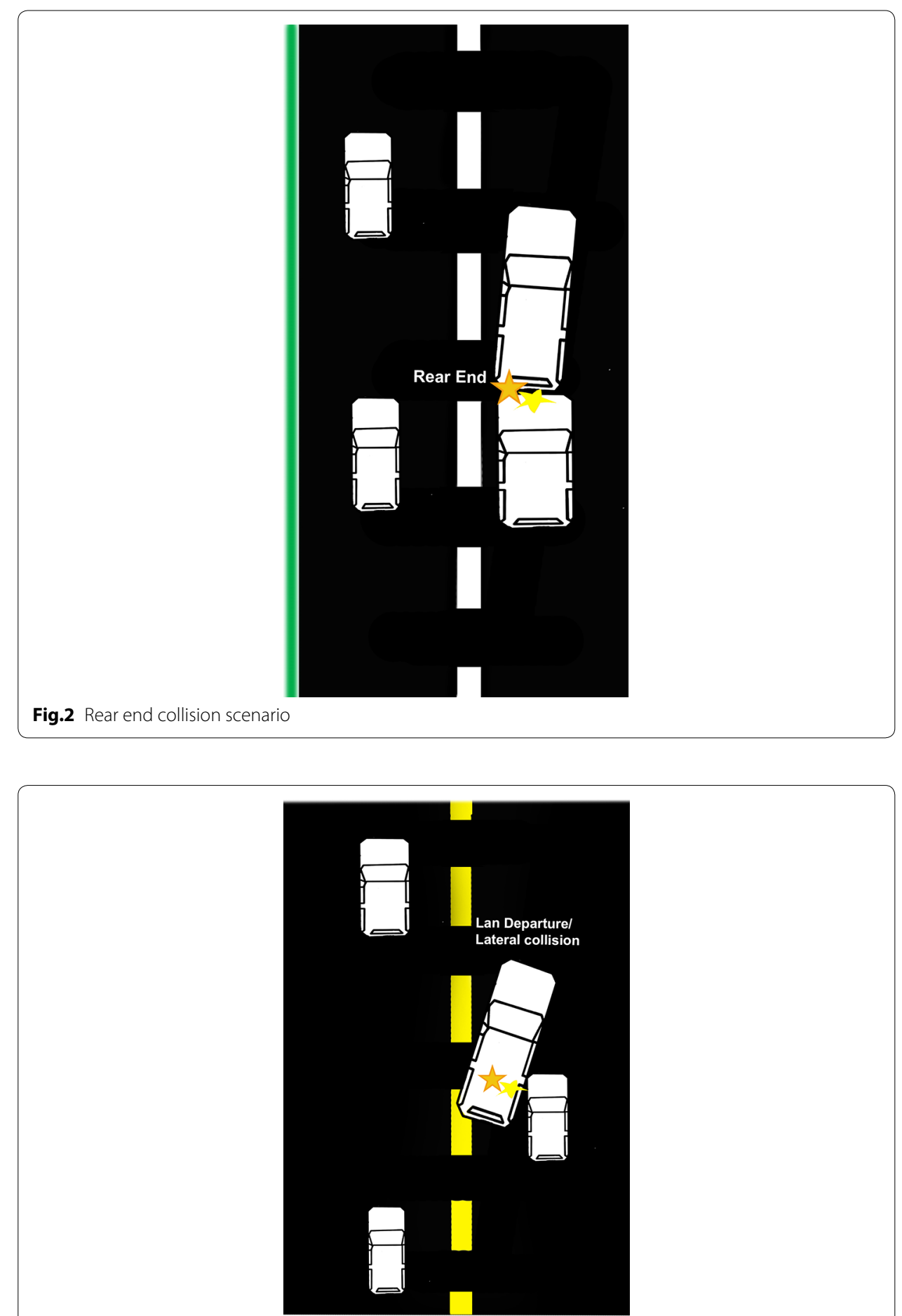

Fig.3 Lateral /lane departure/blind spot collision scenario

The lateral collision scenario is shown in Fig. 3. According to Wegman (2004) head-on or T-Bone collisions are the main reason for $60 \%$ of all deadly collisions in economic cooperation and development (OECD) member countries. According to Rosey et al. (2008), head-on and intersection collisions contribute as $80 \%$ of fatal collisions leading to the deaths and injuries in rural areas of Europe. According to National Highway Traffic Safety 
Administration (NHTSA), Intersection collisions contribute overall $47 \%$ of all vehicle collisions in the United States in 2010 (Traffic safety facts 2010). According to Wachtel and Lewiston (1994), 64 \% of bicycle-motor vehicle accidents occur over intersections. Intersection collisions are considered as most typical collisions happened with old drivers.

\section{B. Location based}

In this section, location based road collisions are described. Intersection/T-bone collisions falls in location based collisions. We have given the definition of intersection/Tbone collisions following its pictorial illustration and statistics.

\section{a. Intersection/T-bone}

Definition According to Chakraborty et al. (2011), when one vehicle collides in the side of another vehicle in a perpendicular fashion due to the violation of red-light or stop signals at an intersection, it is known as T-bone collision.

The T-bone collision scenario is shown in Fig. 4. The world statistics of intersection/ T-bone collisions are given as follows. According to NHTSA every year 840,000 blind spot accidents happen in the USA causing 300 fatalities. According to Trucks (2015), lane departure accidents are total $10 \%$ of all accidents happened in Europe. According to Benavente et al. (2006), lane departure collisions are total $19 \%$ of all accidents happened in Massachusetts from 2002 to 2004. According to Highway statistics 2013 (2015), only in USA, 5570 and 5345 people died in lateral collisions during the year of 2012 and

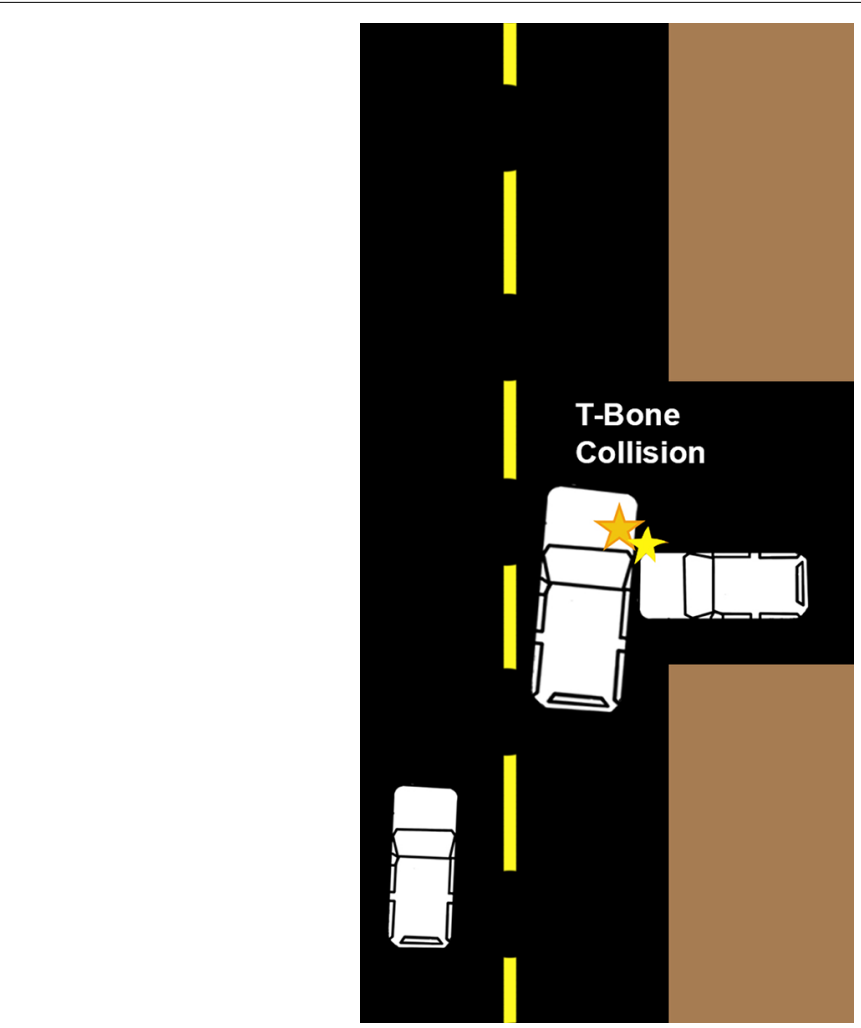

Fig.4 T-Bone collision scenario 
2013 respectively. According to Shasthri et al. (2015), children involved in lateral or side impact collisions have a high death rate than in front side collisions.

\section{Species based}

Species based collisions are divided into two subcategories, i.e. pedestrian and animal. We have presented statistics related to the pedestrian and animal collisions in sections a and $\mathrm{b}$ respectively.

a. Pedestrian-collision statistics Deaths of pedestrians in road collisions are a tragic issue of human society. According to Crocetta et al. (2015), 1.2 million pedestrians die in road collisions annually, of which $35 \%$ are children. According to Bennet and Yiannakoulias (2015), road collisions are the main cause of child pedestrians' death in Canada. According to Tulu et al. (2015), road collisions are the dominant cause of pedestrian deaths in Ethiopia. According to Koopmans et al. (2015), every year in the United States (US), around 900 child pedestrians are killed with an additional 51,000 injured.

b. Animal-collision statistics Animals are also one of the victims of road collisions. According to Loss et al. (2014), collisions between vehicles and animals kill hundreds of millions of birds and other animals each year. According to Rowden et al. (2008), only in Australia more than 11,635 accidents happened between vehicles and animals in the time period of 2001-2005. According to Langbein (2007), 30,500 accidents happened in Brittan between deer and vehicles in the time period of 2000-2005.

\section{CPS}

Different phenomena of this physical world have their effects on humans' lives. As an instance, according to Carod-Artal (2016), health phenomenon like Zika virus affected badly approximately 1.5 million people of Brazil. According to World Health Organization (WHO 2009), the phenomenon of road accidents affects almost 1.2 million people on the yearly basis. According to Hilhorst (2002), natural disaster phenomenon like earthquake affected about 8 million people in Nepal. In the light of the above studies, there is a need to have such mechanisms, which makes this world a better place to live by minimizing the effects of these phenomena.

Cyber-physical systems may help to make physical world a better place to live. As reported by Lee et al. (2010), CPS can help to solve the grand challenges of transportation, healthcare, manufacturing, and energy. By integrating computing devices with internet, noted by Baheti and Gill (2011), affects of global warming can be minimized. According to Lee (2008), the quality of human lives can be improved by adapting CPS related applications. Hence, the concept of CPS is currently used in different domains to improve their performance.

\section{Health care systems}

CPS has been found suitable to tailor better health care applications for human society. A cloud-based CPS has been proposed by Zhang et al. (2015) for better patient-centric health care. Fuzzy logic based mobile healthcare system has been proposed by Costanzo et al. (2016) to provide better healthcare facilities for older citizens. Loneliness and 
lack of proper care affects the health of senior home alone elders, badly. To overcome this issue an internet of thing (IOT) based health care system known as CyPhyS+ has been proposed by Dagale et al. (2015). From the above discussion, it can be concluded that CPS based health care systems are significantly contributing towards health care issues of human society.

\section{Road safety-autonomous vehicles}

Vehicular CPS has been explored as one of the solutions to improve the road safety. Abid et al. (2011) have proposed in-car vehicular cyber-physical system (VCPS) using vehicle2-vehicle communication to enhance the road safety. In another research study Huang et al. (2016) have proposed lane departure and forward collision warning to improve road safety by warning the drivers affected by high fatigue factor. According to Mutz et al. (2016) road safety can be improved by autonomous vehicles based VCPS. However, Autonomous vehicles based VCPS are least explored.

Autonomous vehicles are of many types. Kok et al. (2013) have proposed unmanned arial vehicle (UAV) helicopter, which can perform its path planning autonomously. Motwani et al. (2013) have proposed underwater autonomous vehicle for mine sweeping and harbor protection purposes. In Sailan and Kuhnert (2015), a novel mobile ground autonomous vehicle known as DORSI robot has been proposed to fulfill the needs of the military. However, in this survey, our primary focus is collision avoidance warning/ avoidance systems using ground-based semi/fully autonomous vehicles.

Autonomous ground vehicles can be divided into a semi and fully autonomous vehicles. In this section, semi and fully autonomous vehicles are discussed.

\section{A. Autonomous ground vehicles}

Autonomous ground vehicles can be very useful to minimize the road traffic problems. For example according to Litman (2014), road congestion issue can be solved by deploying autonomous vehicles. Furthermore, Mersky and Samaras (2016) have proven in their research studies that road traffic pollution may be reduced significantly using autonomous vehicles. Also, according to Riaz et al. (2015a), road collisions can be decreased with the help of autonomous vehicles. However, the role of autonomous vehicles for better road traffic management still needs research efforts.

Table 1 presents the development timeline of ground autonomous vehicles. The initial experiments were started in 1920 with Achen Motor Company. First truly autonomous car had been realized in 1984 by ALV labs. Then in 1987, Mercedes Benz started work on its first autonomous car. Google developed the first state of the art, modified Toyota Prius, ground autonomous vehicle in 2010. In addition, Google developed a twoseated autonomous car in 2014 and it is expected to have its driving license by 2017. Furthermore, many autonomous car prototypes have been developed in 2013 by different automakers like Ford, Toyota and Nissan.

a. Experimental-state of the art by automakers

- BMW

BMW automaker company built its first autonomous car in 2014 as shown in Fig. 5a (Goodrich 2013). To perform the collision avoidance, BMW AV is equipped with vision system using cameras, light detection and ranging (LIDAR) system, $360^{\circ}$ radar, 
Table 1 Ground autonomous vehicles development timeline

\begin{tabular}{lll}
\hline Time line & Organization/automaker & Reference \\
\hline $1920-1950$ & Achen Motor Company & Anderson et al. (2014) \\
1980 & Univ. Bundeswehr Munich & Cord (2009) \\
1984 & ALV & Cord (2009) \\
1987 & Mercedes Benz & Cord (2009) \\
1994 & Univ. Bundeswehr Mnchen & Dickmanns et al. (1994) \\
1998 & University of Pavia & Broggi et al. (1999) \\
2007 & Braunschweig University, Berlin University & Rauskolb et al. (2008) \\
2010 & Google Car & Markoff (2010) \\
2012 & Stanford University & Funke et al. (2012) \\
2013 & Ford, Toyota Lexus, Nissan & Lari et al. (2014) \\
2014 & R\&D, Audi AG & Fleming (2015) \\
2014 & Google car & Manawadu et al. (2015) \\
\hline
\end{tabular}

and ultrasonic sensors. BMW's AV has been tested with its autonomous driving capabilities over 9000 miles.

- Audi

Audi built an AV having the capability of piloted driving (Payre et al. 2014). Using piloted driving feature it can monitor the status of drivers and can avoid collisions caused by impaired driving. It has been tested successfully in heavy traffic with the speed of $40 \mathrm{mph}$. To avoid the collision differential GPS and 3D cameras are installed. The Audi autonomous car has been shown in Fig. 5b.

- Ford

In 2013, Ford introduced automated fusion hybrid autonomous vehicle as shown in Fig. 5c (Lari et al. 2014). It is equipped with LIDAR to avoid the collisions by sensing its surroundings. In collaboration with Massachusetts Institute of Technology (MIT), advanced algorithm has been used to predict the future position of vehicle and pedestrian, which helps in avoiding the AV-pedestrian collisions more efficiently.

- Toyota-lexus

Toyota presented its first autonomous car prototype at the annual "Consumer Electronics Show” (CES) 2013 in Las Vegas (Meinel 2014). Its active safety system uses laser tracking, stereo cameras, GPS, and mm-wave radar to avoid the road collisions. In the case of any road collision, it has rescue and response system as well. The AV has the capability to distinguish between different colors of traffic light signals and can measure the trajectory of another vehicle on the road for safe path planning. The Toyota-lexus AV is shown in Fig. 5d.

- Nissan

Nissan introduced its AV Infiniti Q50, shown in Fig. 5e, in 2013 (Bimbraw 2015). It uses cameras, radar, and other next generation technology to avoid the collisions. The model delivers various features like lane keeping, collision avoidance, and cruise control. It was the first car equipped with virtual steering column. The driver need not manually operate the accelerator, brakes or steering wheels.

- Google

Google a non-automaker company presented its latest two seats autonomous car in 2014 as shown in Fig. $5 f$ (Fleming 2015). The toy-like concept vehicle has two seats, a 


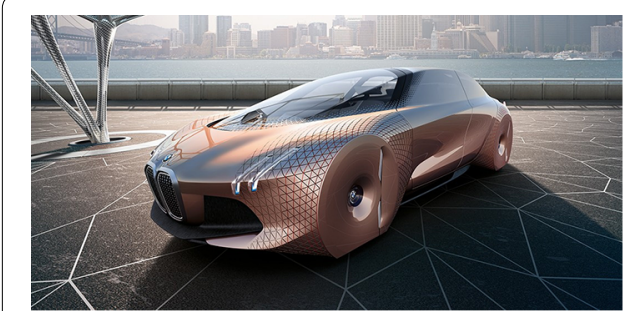

a

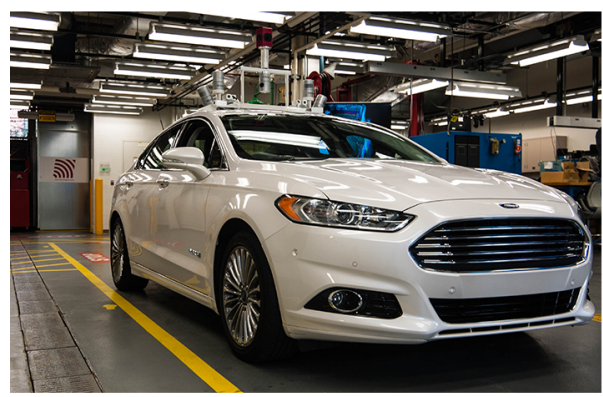

C

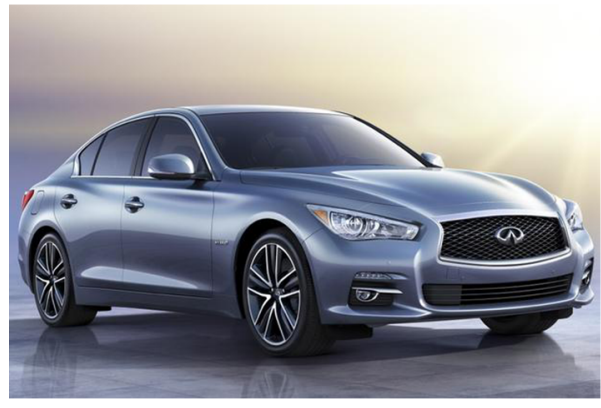

e

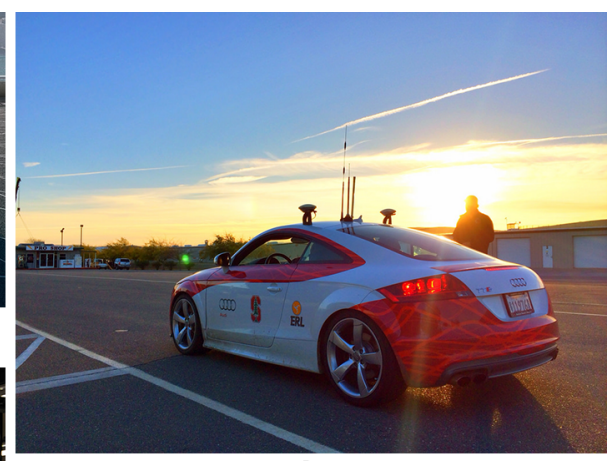

b

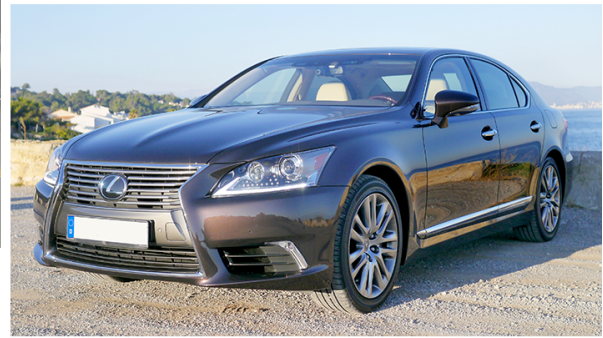

d

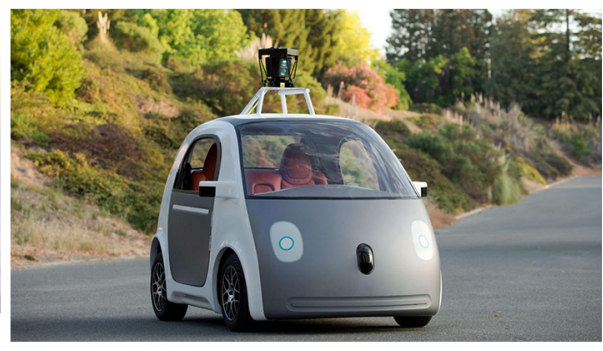

f

Fig. 5 State of the art autonomous cars (Note: Figures $5 \mathrm{a}$ to $5 \mathrm{f}$ are used as they are available online under the "Free to use and modify"title). a BMW Autonomous car (Photo credit: NJSTOKES URL: http://www. rgscomputing.com/2016/05/13/bmw-will-launch-its-first-self-driving-car-in-2021/), b Audi autonomous car (Photo credit: Joseph Thornton URL: http://www.flickr.com/photos/jtjdt/11061195763), c Ford automated fusion hybrid autonomous vehicle (Photo credit: Jonathan M. Gitlin URL: http://www.arstechnica.com/ cars/2015/08/face-to-face-with-fords-self-driving-fusion-hybrid-research-vehicles/), d Toyota-Lexus advanced active safety research vehicle (Photo credit: Alexander Stoklosa URL: http://www.blog.caranddriver.com/ autonomous-lexus-advanced-active-safety-research-vehicle-revealed-detailed/), e Nissan infiniti Q50 autonomous car (Photo credit: Basem Wasef URL: http://www.autotrader.com/car-reviews/2014-infiniti-q50-firstdrive-review-215728), f Google autonomous car prototype (Photo credit: Parker Wilhelm URL: http://www. techradar.com/news/car-tech/google-s-self-driving-cars-get-3-million-miles-of-practice-a-day-1314251)

screen displaying the route and a top speed of $25 \mathrm{mph}(40 \mathrm{~km} / \mathrm{h})$. An array of sensors allows the vehicle's computer to determine its location and surroundings and it can "see" several hundred meters, according to Google.

b. Experimental-state of the art by academia Vision system helps autonomous cars in detecting, like human drivers, incoming road terrains and obstacles. However, lane markers detection over curved road is still a challenging task. To overcome this issue, Al-Zaher et al. (2012) have carried out both the land and obstacle detection by introducing a vision system in the autonomous vehicle. The vision system consists of a low-cost webcam with 
$320 \times 240$ pixels and has the ability to monitor the front vision of the autonomous vehicle. The research is based on a technique for using calibrated cameras to detect obstacles with vision sensors. Lanes are marked by white line marks, which can be identified and captured by the webcam. The numerical simulation has been carried out under the MATLAB/Simulink environment. The missing part of research is that the appearance of sudden obstacles likes pedestrians and animals have not been considered, and may cause severe collisions. These types of road tweaks can be handled using some proactive approach. In this regard, human emotions might be helpful in proposing, as emotions enhance the vision system of proactive human driver, better land and obstacle detection schemes.

Road dynamics are of a complex nature, any sudden tweak in traffic dynamics can lead towards a dangerous road accident. Hence, there is a need of real time collision avoidance techniques, which help the autonomous car to safe its passengers from any potential harm. Park (2008) has proposed a real-time collision avoidance by fusing potential field method (PFM) and vector field histogram (VFH) for unmanned ground vehicles. Furthermore, the concept of steering, obstacle, and integrated force fields are proposed by extending PFM and VFH. The autonomous navigation system is responsible for generating steering force, laser range finder of autonomous vehicle generates the obstacle force field, and using the integrated force field overlapped these two fields, modified steering, velocity and emergency stop commands are created to avoid collision. The experimental autonomous vehicle (XAV) is composed of a stereo camera, 2-axis actuator, and a computer. The proposed method is not only capable of avoiding collisions from stationary obstacles like a cylinder and barriers, but also from pedestrians and moving vehicles. The missing part of research is the lack of a cognitive agent, to act like central entity, which compute steering and obstacle forces and issue emergency stop commands. In this regard, any Agent based Modeling paradigm can be explored to enhance the efficiency of the proposed system.

If the following drivers have some mechanism to get pre-accident alerts, using some gadgets and communication system, chained accidents can be avoided. However, alerting the following drivers on real time is a challenging task. Chen et al. (2012) have proposed a portable graphical user interface enabled GPS based collision detection and alerting test bed. The proposed test bed consisted of free scale 9s12XEP100 16-bit HCS12X SPU with 512KB flash EEPROM and 32KB RAM. To perform vehicle-2-vehicle (V2V) communication Ralink RT 2500 WLAN card has been used. The system helps the drivers to monitor the possible collision from the neighboring vehicles. Using this information, the driver can send alert messages to the neighboring vehicles. The major drawback of proposed system is that it is using graphics based driver warning system. Whereas, according to Riaz et al. (2013), graphics based alert system can distract the driver attention and it might cause a road accident.

The overview of collision detection hardware used by above mentioned state of the art ground autonomous vehicles is presented in Table 2.

Most of the collisions avoidance solutions of autonomous vehicles, interesting to note, are inspired from the other fields like economics (game theory), psychology, nature, and physics. Hence, this synergistic approach helps the researcher to devise novel solutions. For example, in order to avoid the road collisions between autonomous vehicles, game 
Table 2 Overview of state of the art ground autonomous vehicles

\begin{tabular}{llll}
\hline S. no & State of the art & Collision detection hardware & Reference \\
\hline 1 & Automaker-BMW & $\begin{array}{c}\text { Vision system using cameras, LIDAR system, 360 radar, } \\
\text { and ultrasonic sensors }\end{array}$ & Goodrich (2013) \\
2 & Automaker-Audi & Differential GPS and 3D cameras & Payre et al. (2014) \\
3 & Automaker-Ford & LIDAR & Lari et al. (2014) \\
4 & Automaker-Toyota-Lexus & Laser tracking, stereo cameras, GPS and mm-wave radar & Meinel (2014) \\
5 & Automaker-Nissan & Cameras, radar & Bimbraw (2015) \\
6 & Non-automaker-Google & LIDAR, array of sensors & Fleming (2015) \\
7 & Academia-Al-Zaher et al. & Low-cost webcam with 320 $\times$ 240 pixels & Al-Zaher et al. (2012) \\
8 & Academia-Choe et al. & Stereo camera, 2-axis actuator, and a computer & Park (2008) \\
9 & Academia-Chen et al. & GPS & Chen et al. (2012) \\
\hline
\end{tabular}

theory and cellular automata inspired solution has been proposed in Rane et al. (2014). Employing the proposed technique, autonomous vehicles can reach to their destinations in minimum time with the least number of collisions.

Autopilot system of an autonomous car, make decisions like collision avoidance, path planning, and route optimization, act like a human driver. Hence, it would be interesting to design it after human drivers' mental functions and behaviors. Czubenko et al. (2015) have proposed a model of intelligent system decision making (ISD) based on human psychology for autonomous vehicle driving. The concepts of need and reaction have been integrated with ISD system. The proposed system functions satisfactorily to achieve its goals. However, human emotions, which are important part of human psychology, have been ignored in making driving decisions.

Cooperative autonomous driving is very useful, by building cooperative vehicular networks, in avoiding road collisions. However, building an efficient autonomous lanedriving algorithm is a difficult task due to high speed and unpredictable maneuvers of neighboring autonomous vehicles. In this regard, Lamia Iftekhar and Olfati-Saber (2012) have proposed a flock inspired lane-driving algorithm to keep the vehicle moving along the mid lane without colliding with neighboring autonomous vehicles. Further, a novel path-planning model has been presented for the better operation of autonomous vehicles in the absence of speed lanes (Kala and Warwick 2013). The technique of lateral potential is used to solve this problem. The potential for a vehicle include obstacles, road boundaries and all sides of the vehicle. However, it would be interesting to model the proposed flock inspired lane-driving algorithm by envisioning each autonomous vehicle as a separate cognitive agent. The agent-based model might be helpful in exploring interaction dynamics between neighboring autonomous vehicles and in designing better cooperative lane-driving algorithm.

Vehicles have different types of actuators like brakes, steering, and gas pedal, which are used to avoid road collisions. To avoid the road collisions, human drivers use brakes more frequently as compared to steering actuator. Whereas more optimal maneuvers can be performed using only steering. Llorca et al. (2011) have proposed fuzzy logic based autonomous vehicle-to-pedestrian crash prevention system, which uses steering as a basic actuator to avoid the collisions. To detect the pedestrians ahead, a stereo vision sensor has been utilized. A fuzzy controller has been used for the execution of the collision avoidance maneuvers. Lateral displacement and the actual speed of the vehicle 
have been used as inputs to the fuzzy controller, whereas steering position is treated as its output. The parameters used to avoid collisions from pedestrians have been defined after the brief study of the human drivers. However, human emotions are ignored while designing the so-called pedestrian collision avoidance system.

In Kraus et al. (2009), the operational capabilities of the ground autonomous vehicle have been improved by introducing emotions. Driving issues, including accidents, time efficiency, and avoidance of close gaps have been resolved by implementing emotion based cognitive appraisal model. The solution has been provided in the graphical and mathematical form. The presented model is tested by simulating the two merging lanes where emotions inspired autonomous vehicles have to avoid accidents. However, the performance of the model has not been evaluated for any of the collision types like the rear end, front end, or lateral one. Furthermore, it would be better to model the autonomous cars as emotional cognitive agent using some agent based modeling (ABM) paradigm. It might be helpful in exploring the interaction patterns and emerging emotions between autonomous cars. In addition, autonomous cars might be modeled as social agents, by building their artificial societies, and then exploring the role of emotions in their interactions.

\section{B. Semi-autonomous ground vehicles}

Definition According to NHTSA "Vehicles at this level of automation enable the driver to cede full control of all safety-critical functions under certain traffic or environmental conditions and in those conditions to rely heavily on the vehicle to monitor for changes in those conditions requiring transition back to driver control. The driver is expected to be available for occasional control, but with sufficiently comfortable transition time" (Administration NHTS 2013).

Road safety can be improved by providing some driving assistance by vehicles. According to Anderson et al. (2009) semi-autonomous cars can avoid road collisions by alerting the driver. According to Bengler et al. (2014) semi-autonomous vehicles can ensure a collision-free traffic environment by assisting the driver through sound alerts, speed automation, safety lane changing mechanism, automatic braking system, and parking assistance system. According to Daza et al. (2014), a real time drowsiness detection system of a semi-autonomous car can avoid collisions by sending alerts to the distracted driver.

a. Semi-autonomous car: rear end collision warning Active road safety systems help in avoiding the road collisions, when human drivers last their control due to drowsiness, use of alcohol, and other in-vehicle activities, by keeping the vehicle in control. The performance of active safety systems, employing machine-learning algorithms, which help them to predict the future state of the vehicle, can be increased. An et al. (2014) have proposed a linear discrimination analysis (LDA) based rear end collision warning system. LDA has been utilized because it can perform data classification and maintains information about the class of the training data, unlike the principle component analysis (PCA). In the proposed model, the vehicle state and TTC has been used as feature space and additional feature respectively. The alarm is activated $1.86 \mathrm{~s}$ before the occurrence of rear end crash, which is enough to perform a safety maneuver by the human driver. Although, 
the authors have used vehicle state and TTC parameters to predict the rear end collisions, they have missed human mental state and its current/future emotional state in this regard. Vehicles are driven by human drivers, which are cognitive as well as emotional in nature. Current mental and emotional state of the human driver plays an important role in the future state of the vehicle. It would be interesting to include human mental and emotional state along with vehicle state and TTC parameters to train LDA for better performance. Furthermore, drivers have been alerted $1.86 \mathrm{~s}$ before the accident, what if the drivers are mentally distracted and do not take any action. In this case, there is a need of some mechanism that takes action autonomously to avoid the collision.

Semi-autonomous vehicles are equipped with radars and cameras, which help them to predict the chances of a rear-end collision. However, in bad weather, snow or fog, radar and cameras can perform false predictions. With the advent of $\mathrm{V} 2 \mathrm{~V}$ communication, collisions can be predicted more efficiently in all types of weather. Li et al. (2014a) have proposed V2V communication based rear end CAS. A risk perception based car following model is used to avoid the rear end crashes. The necessary data about vehicle includes velocity, position, and acceleration value is gathered. After collecting the required data, the leading vehicle is determined. It is done with the help of GPS and then the decision-making analysis is performed to avoid the collision. The drawback of this research is that risk perception, which is an emerging product of human cognition and emotions, is used without considering driver emotions.

Dedicated short-range communication (DSRC) is a short-range wireless system that helps the vehicles to communicate with each other. However, the performance of DSRC based rear end collision warning systems suffers due to uncertain measurement errors and cause high rates of false alarms. Xiang et al. (2014) have proposed a rear end crash warning system using dedicated short range communication (DSRC) based in-expensive high-end devices. The new model proposed in this paper is based on neural networks (NNs). Through training and validation, the $\mathrm{NN}$ model is able to provide emergency warnings with an improved performance of false alarm probability under $20 \%$ as compared to the $70 \%$ previously. The drawback of research is vehicles are using DSRC protocol, which has been proved inefficient in congested highways.

Fuzzy logic has been found useful to model the human assistance systems as compared to the classic approach. However, the question is fuzzy logic can be utilized to design automatic collision avoidance systems (CAS). Milanés et al. (2012b) have proposed fuzzy logic inspired rear-end CAS in the busy traffic situations. Two fuzzy controllers are proposed to achieve efficient system. The collision warning system (CWS) fuzzy controller is used to generate the warning of the crash for the driver. The second fuzzy controller collision avoidance system (CAS) is used to perform the necessary maneuvers to prevent the crash. The important inputs, which are provided to the system, are speed and the displacement required to perform the maneuver safely. The system is tested in the real environments and the results are satisfying and encouraging. In this system, the only braking action of the preceding vehicle has been considered. The steering actions are neglected and considered as a future work.

Humans are cognitive and use their cognition, crisis index one of cognitive feature, to predict the emergency degree of a possible collision. This human capability of emergency prediction can be used to design efficient rear end collision avoidance techniques. 
Previously, many autonomous controllers have been designed, by keeping in mind vehicle sensors, while ignoring the driver's state, like emergency degree or driver's cognition of crisis state of road condition, to avoid the rear end collisions. Computing crisis index through standard mathematical equations is a difficult task, and in this regard, fuzzy logic has been found a suitable tool. Li et al. (2014b) have presented a fuzzy logic based control strategy to avoid the rear end crashes. A crisis index table, representing driving conditions, is presented to know about the chances of the crash with the following vehicle. The factors of the crises are crisis state, the relative distance of vehicles and the speed of the following vehicle. According to the authors, it is not possible to calculate the crises index through a mathematical equation, so fuzzy logic is used for this purpose. The proposed fuzzy controller uses the Mamdani min-max function. The simulation shows the validation of the proposed algorithm. The missing part of research is that crises index has been generated without considering driver emotional state, whereas the emotional state of the driver plays an important role in this regard. For example, in the state of fear, the attention level of drivers is high and they apply the brakes more consciously as compared to the state of anger, where they do not care about the safety distance and violate the safety rules.

b. Semi-autonomous car: rear end collision avoidance Rear end collisions can be avoided, specifically for freeways buses, by generating in-time collision warnings. However, the problem is to determine, most suitable time, to initiate these warnings. In this regard, rear end collision avoidance scenarios have been studied by Chang and Chou (2009) using a simulator with the emergency braking approach. Data regarding the behaviors of different bus drivers towards the rear end collision warning system has been collected using a simulator. The authors have found that different subjects have different driving behavior, reaction time, and deceleration rate. The collected data is then used to determine in-time alert initiation conditions. The missing part is that the rear end CAS is designed by keeping in mind the cognition of human driver but ignoring its emotional aspects. Because, emotions play an important role, noted by Riaz et al. (2013), in making collision avoidance decisions by human drivers.

Emergency steering assistance (ESA) can play an important role in rear end collision avoidance. However, the disadvantage of emergency steering is that the maneuver is more difficult for the average driver. Therefore, an advanced driver assistance system is required. To fulfill this requirement, a driver assistance path planning algorithm using fifth order polynomial has been proposed by Keller et al. (2014). The authors claimed that there are three phases of the emergency steering actions: the first phase guides the vehicle to the appropriate path. The second phase involves the over steering phase, which depends on the situation. The third and final phase is the guidance of the vehicle to the right lane. The results of the simulation are satisfactory. The role of human emotions has been ignored in decision making of emergency steering while emotions have heavy effects on driver's decision-making during emergencies. Table 3 presents the summary of semi-autonomous car based rear end collision warning/avoidance systems.

c. Semi-autonomous car: blind spot/lane departure/lateral collision warning Blind spots are the most vulnerable places for collisions because drivers cannot judge the presence 


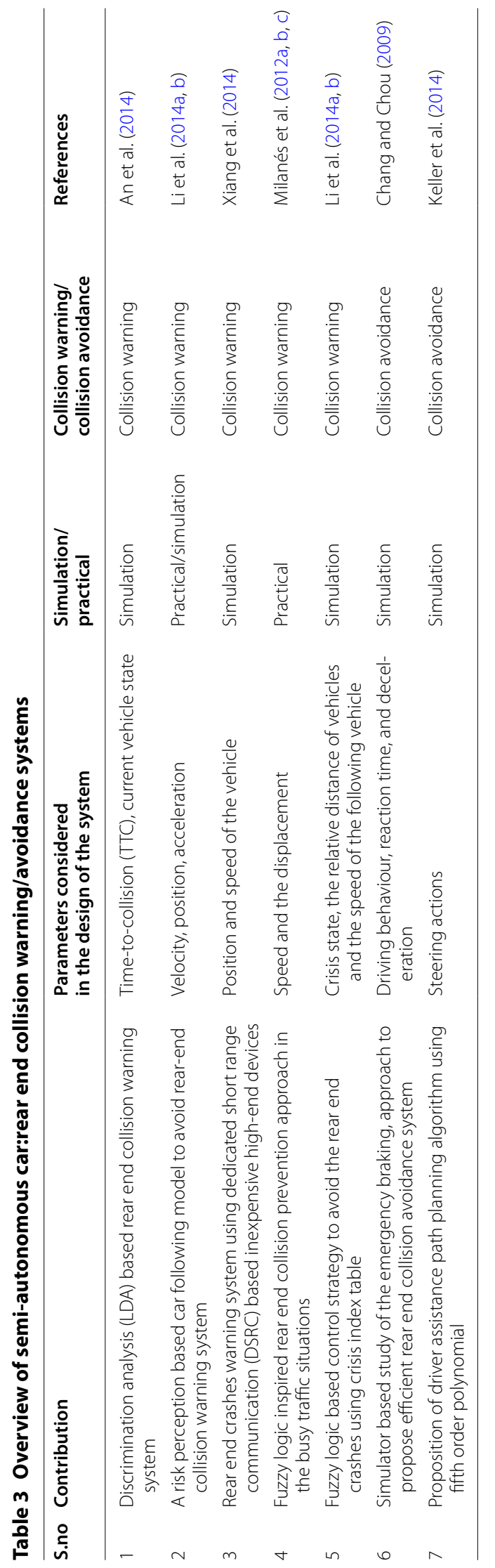


of another vehicle or road hazard through their naked eye. Hence, there is a need of such mechanism, which detects the presence of road hazard in the blind sport and generates in-time warnings to the diver. In this regard a blind spot collision avoidance mechanism has been presented by Uselmann and Uselmann (2004). The proposed system helps the drivers to avoid collisions from out of the vision hazards. The sonar device is connected with the microprocessor and it emits a sound wave in the blind spot to detect the obstacle. To receive the reflection of the emitted wave, the sonar has the receptor. The display panel is mounted inside the vehicle. Whenever the sonar detects any obstacle, it is displayed on display panel to alert the driver. In this way, driver gets the ability to avoid blind-spot collision. The drawback of this research is usage of sonar device, which might be failed in bad weather like snow or fog. Hence, it would be better to explore the same approach using vehicle-2-vehicle (V2V) communication for the detection of road hazards in blind spots.

King (2004) have proposed a polarametric blind spot detection mechanism. The presented system is using circularly polarized transmitters and recipients approach. This system provides the correct information about the objects present in the blind spot and its cost is low. The antenna mounted on the vehicle emits the signal and receives the reflection of the circular signals. In this way, it identifies the existence of another vehicle. When the system detects the presence of another vehicle, it alerts the driver about it by generating a visual signal. It also warns the nearby vehicles, about detected road hazard, using a light signal.

In Schwindt et al. (2015), a lane departure warning system has been presented. The modules, which are utilized in this research, include left and right rear sensors, forward sensor, direction sensor, processing unit, memory, and $\mathrm{I} \backslash \mathrm{O}$ interface. This system uses the front sensor to check the lane location of the vehicle and tracks the vehicle coming from the opposite side. The sensors present at the sides of the vehicle take care of the vehicle moving parallel or overtaking vehicles. The information from both types of sensors is then given to the $\mathrm{I} \backslash \mathrm{O}$ interface. It integrates the information and forwards it to the processing unit. Where the decisions about the lane keeping and lane departure are taken and warnings are generated accordingly.

A blind spot warning (BSW) system using hepatic feedback approach has been proposed by Chun et al. (2013). The warnings are provided to the driver using a seat belt or the steering wheel of the vehicle. These warnings are initiated only if during lane changing there is the possibility of the crash with another vehicle. This system was tested using the human drivers on a simulator and both seat belt and steering wheel warnings were utilized. The drivers were divided into two age groups, young and old drivers. The hepatic warnings through steering wheel are found more effective than seat belts.

Kusano and Gabler (2012) have presented a computational model of road departure crashes. The model is developed by collecting the data from the real world collisions. A simulation is also designed, which is based on this data to show the effectiveness of the lane departure warning (LDW) system. This model is used to make the simulations of collision of the vehicle with the objects near the road. It is stated that the crashes can be avoided by performing a small steering or braking maneuver. The simulation shows that if the driver gets the LDW when departing the road, then nearly $5 \%$ of collisions can be avoided. 
Huang et al. (2015) have proposed pulse steering torque warning system to overcome the lane departure accidents. A model of the lane departure warning system is designed which include 12 factors. For the experiments, the data are collected from twenty drivers who drove in different lane departure situations. The experiments show that steering wheel with asymmetric pulse torque can get the right reaction of the driver. For the lane departure, the large pulse torque is better than the small amplitude pulse.

Jeffrey et al. (2015) have studied the protection advantages of two different on-board safety systems (OSS), LDW and roll stability control (RSC), which are installed on trucks. The data are gathered from different participating carriers. The results of the study show the great benefits of using both OSS. The numbers of crashes of trucks with these systems are compared with those not having the proposed system. The results show that the accident ratio of the trucks with LDW is 1.917 \% less than the accident ratio of the trucks without LDW.

d. Semi-autonomous car: blind spot/lane departure/lateral collision avoidance Overtaking is a complex driving maneuver and involves high risk of collision if performed carelessly. An autonomous overtaking system can make, by avoiding the possibility of human driver distraction, overtaking a safe maneuver. The cognitive automatic overtaking system has been proposed to avoid the lateral collisions during overtaking maneuvers by Milanés et al. (2012a). The vision system is used to find out the preceding vehicle's speed and width. Using speed and width parameters, the system determines the length of the preceding vehicle and adjusts the speed of the following vehicle accordingly, in order to minimize the overtaking time. The fuzzy controller is also utilized to perform the steering maneuver automatically. The inputs of the fuzzy controller include speed, the width of the preceding vehicle, and lateral displacement signals obtained from the vision system. The missing part of research is that human decisions involve different types of emotions, fear and sympathy, as well during overtaking the different types of vehicles like, truck, car and motorbike. For example, the lateral safety distance and speed are greater and low respectively, as a human driver feels high fear during overtaking truck due to its size, when a car overtakes the truck. In another case the same driver has small lateral distance and high speed, as the level of fear is very low, when to overtake a cycle.

Unintended lane departure is one of the main causes of highway accidents. These collisions can be avoided by automating the process of lane departure, which has the capability to perform in-time steering action. To address this issue, a lane keeping assistance system has been proposed to prevent the unintentional road departure by Benine-Neto et al. (2014). The proposed system is based on a state feedback dynamic controller. Some objectives are also defined for the controller, steering assistance activation law, and computation of the control law. The effectiveness of the control strategy is evaluated through a simulation, which is designed with CarSim environment. The system is further tested on a real world model vehicle. The results of the simulation are satisfying in terms of safe lane departure. However, the performance of the system might be improved by modeling the proposed system as a cognitive agent. Although, authors have performed the assessment of driver's awareness before handing over the steering control, they have missed the assessment of emotional state of the driver. Emotions play an important role in making lane departure decision. For example, an angry driver will perform more risky lane 
departure maneuvers as compared to the normal one. Hence, it would be interesting to add driver's emotional state assessment along with attention assessment, which restricts the steering control, if the driver is in negative emotion.

In another research work road departure prevention (RDP) system has been proposed by Katzourakis et al. (2014). The RDP system is used when the vehicle is about to leave the road. For the emergency steering actions, the developed RDP system was evaluated under four scenarios. In the first scenario, the RDP was inactive and driver dealt with the whole situation. In the second hepatic feedback (HF) situation, the RDP was activated to assist the driver. In the third drive by wire (DBW) scenario, the level of automation was high. The fourth scenario combined DBW and HF; it combined the properties of both RDP and HF. An X-car driving simulator was utilized to test the system. It is concluded from the results that the DBW based RDP system is better to prevent the road departure of the vehicles and can increase the safety.

Gray et al. (2012) have proposed a safety controller for the prevention of the unintentional lane departure system. The system keeps track of lane departure. The situations in which vehicle leaves the road without knowledge of the driver, the safety system prevents the situation by steering or braking maneuvers. A mathematical model of the system is also proposed. Four types of scenarios were taken into account and experiments were performed. The simulated results show that the proposed controller is capable of noticing and preventing the unintentional road departure during driving.

Table 4 presents summary of semi-autonomous car based blind spot/lane departure/ lateral collision warning/avoidance systems

e. Semi-autonomous car: intersection/T-bone collision warning Intersections are vulnerable to the collisions due to chaotic traffic flow and distracted drivers' behavior. An agent model having communication capabilities can be helpful in decreasing intersection collisions. In this regard, agent based intersection CAS using vehicle-2-infrastructure communication has been proposed by Lee et al. (2013). The system consists of three main agents: intersection, infrastructure controller, and vehicle agents. When a vehicle enters the intersection, then the roadside units notify the infrastructure controller. The vehicle agent and infrastructure controller give the status information to the intersection agent. This information is evaluated and a warning is generated if the risk of an accident is high. The proposed system is developed using java agent development (JADE) environment. The missing part of research is that proper agent based modeling paradigm has not been followed to design the proposed system. Furthermore, the proper agent architecture has not been proposed. In addition, non-standard agent simulation software is used for the development and testing of the proposed technique.

Finding feasible metrics, for the evaluation of the usefulness of intelligent transportation systems (ITSs), in terms of safety is one of the main challenges in the study of vehicular networks. To overcome this issue an intersection collision avoidance protocol has been proposed by Joerer et al. (2014). The collision probability metric has been used to compute the chances of accidents. In this approach, two vehicles approaching an intersection communicate with each other by sending Beacon messages. For inter-vehicle communication (IVC), IEEE 802.11p protocol is employed. The main contribution of this paper includes collision probability estimation along with its integration into a road 


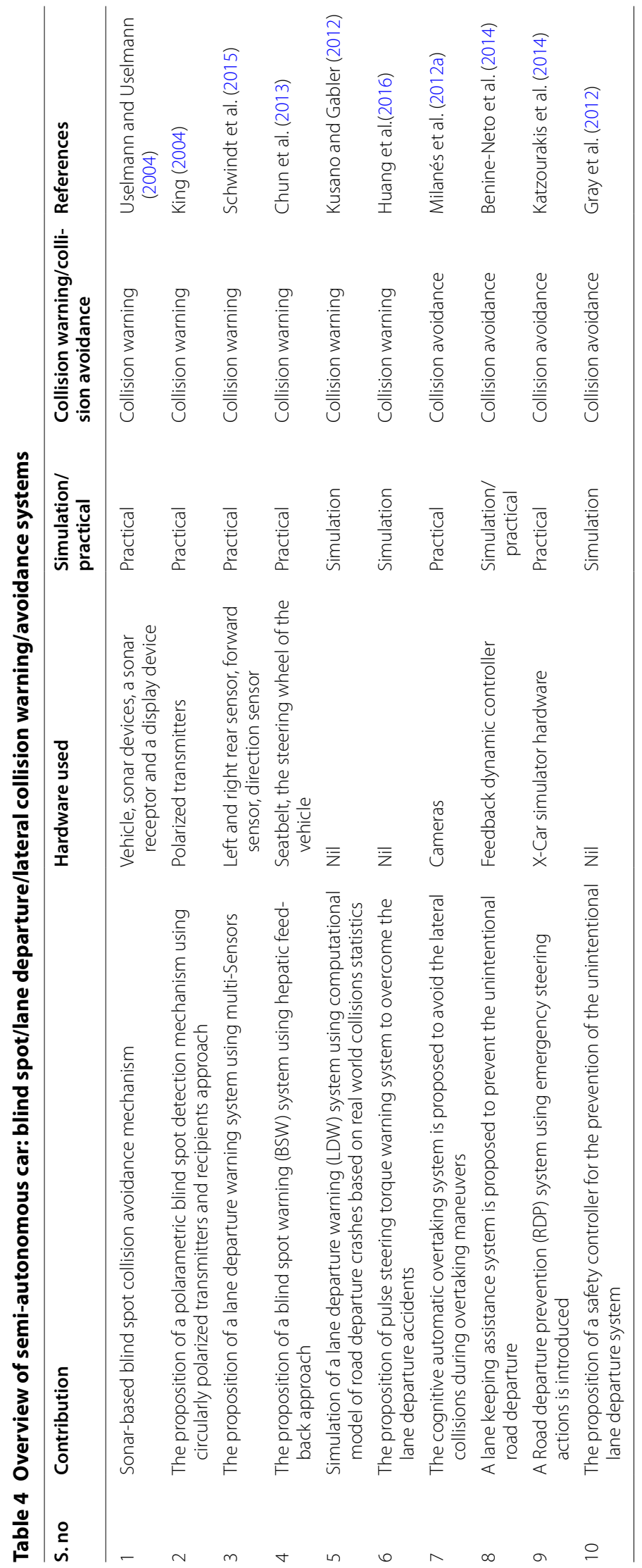


traffic simulation, validation of the probability estimation, and its implication on vehicular networking. This approach can also be used, as claimed by the authors, to compute the possibility of a lateral collision between vehicles.

To avoid collisions over non-signalized intersections, Liu et al. (2012) have proposed vehicle collision warning system based on the concept of internet-connected vehicles. Vehicles are equipped with communication modules to let the vehicles share their information such as velocity, position, and heading, etc. Collision warning system alerts and assists the driver to avoid the collision. For the implementation of the collision-warning algorithm, MATLAB/SIMULINK has been used.

von Eichhorn et al. (2013) have proposed a driver assistance system (DAS) using the $\mathrm{V} 2 \mathrm{~V}$ communication system to avoid the intersection collisions. To perform V2V communication dedicated short range communication (DSRC) IEEE 802.11p is employed. Furthermore, a warning algorithm has been proposed which uses time-to-warn (TTW) parameter to compute the estimated reaction time of the driver. The driver is warned only when the reaction time exceeds a certain threshold.

According to Basma et al. (2011), the collision rate of vehicles is very high over intersections instead of the latest innovations in the field of vehicle safety. To overcome this issue, an intersection collision avoidance (ICA) system using vehicle-2-road (V2R) communication based on Radiotronix Wi.232DTS radio transceiver is proposed by Basma et al. (2011). The radio transceiver operates over the $902-928 \mathrm{MHz}$ public frequency. It is an infrastructure-based system. This system consists of base station (BS) and wireless sensor nodes (WSN). It helps in detecting the approaching traffic and detects if the collision may occur between the vehicles. It then warns the vehicle of high collision probability. A bit error rate (BER) check was performed on the transceivers and comparisons of performance between selected WSN distances were made. For the verification of the system's accuracy, the performance of the overall system was tested as well.

Dabbour and Easa (2014) have proposed an early CAS for semi-controlled urban intersection using radar sensor. The proposed system uses the radar sensor to measure the location and speed of the vehicles approaching towards the intersection and generate warnings in case of danger of collision.

Milanés et al. (2012c) have proposed an intelligent vehicle-2-infrastructure (V2I) communication based urban area traffic management system. V2I communication is performed using wireless accesses for vehicular environment (WAVE) IEEE 802.11p standard. In an urban area, different traffic scenarios can co-exist. To evaluate the traffic situation, a fuzzy traffic management system was evaluated. The traffic conditions are evaluated by a control station to prevent collisions along with improved traffic flow. A driving state indicator is sent to the drivers, which guides them to adjust vehicle's direction and speed. The proposed traffic management system is simulated and tested on Simulink (Matlab) with the dynamics of four model vehicles. Later, this system is also tested on a real test track with four vehicles approaching an intersection from different directions.

Tung et al. (2013) have proposed a cluster-based architecture, using long term evolution (LTE) and Wi-Fi technologies, for intersection collision avoidance. The idea has been presented evidently through proper comparison between homogeneous and selected heterogeneous schemes. Wi-Fi and LTE channels are used for intra-cluster and 
inter-cluster communication respectively. The presented algorithm for cluster formation has been claimed as a lightweight clustering algorithm. However proposed algorithm could not clearly justify the fact of being lightweight, if members are not exchanging their location with the cluster head, then it would be too tough for head node to maintain an updated table of its members' location.

The main drawback of the proposed intersection collision avoidance techniques by Joerer et al. (2014), von Eichhorn et al. (2013), Milanés et al. (2012c) and Tung et al. (2013) is that they are using IEEE 802.11p for IVC purpose. IEEE 802.11p protocol is using seven $10 \mathrm{MHz}$ wide channels in the range of $5.9 \mathrm{GHz}$ spectrum. However, it has been found that these dedicated frequency resources fail to solve the problem of bandwidth allocation due to the increasing number of users (vehicles) competing for same channel within the same area (Riaz et al. 2015b). Furthermore, the delay in safety messages should be less than $200 \mathrm{~ms}$ in vehicular adhoc networks (VANETs). Whereas, due to data contention in the control channel of DSRC the safety message delivery time, packets have to be resent many times, exceeds $1000 \mathrm{~ms}$. It means the proposed intersection collision avoidance schemes will not work properly on the highly congested intersection scenario. It would be interesting to evaluate cognitive radio based solutions to overcome this issue.

f. Semi-autonomous car-collision intersection/T-bone detection/avoidance Driver inattention is one of the major reasons of road accidents. Hence, there is a need of such assistance system, which keep them attentive. Many research studies have been performed in this regard. However, most of them just cover solutions for one type of accident, while ignoring other types. Furthermore, only vehicle dynamics are considered in the design of these crash prevention schemes while ignoring the behavior of drivers at large. To overcome this issue, Kim and Jeong (2014) have proposed an efficient T-Bone and rear end collision detection algorithm for common road scenarios by considering driver behavior. Monte Carlo simulation is used to generate crash probability data considering driver behavior and vehicle dynamics. The algorithm is further consists of a tracking algorithm that uses an interactive multiple-model particle filter, and a threat assessment algorithm that estimates crash probabilities data obtained from Monte Carlo simulation is used to detect the possibility of the collision. The proposed algorithm can distinguish between the crash and near miss cases. The algorithm was tested in three different scenarios, e.g. rear-end, cut in and T-bones. Although, the authors have considered the human behavior in the design of algorithm, they have not examined the role of human emotions. Furthermore, the threat assessment algorithm is proposed without considering the cognitive and emotional structures of brain as amygdala and hippocampus are responsible for threat assessment as noted by Riaz et al. (2015a).

Most of the passive safety systems in practice have capability to mitigate the effects of frontal collisions. No passive safety systems are available to cope with the side or T-Bone impacts. Hence, there is a need of such techniques, which help in mitigating the effects of T-Bone collisions. In this regard Chakraborty et al. have performed two research studies in Chakraborty et al. (2011, 2013). In first research, Chakraborty et al. (2011) have examined how a sudden power action, which includes yaw rotation can reduce the chance of a T-bone crash between two vehicles over the intersection. A torque vectoring 
(TV) technology, also known as the left-right torque vectoring has been evaluated. It is applied to the rear wheels of the vehicle to generate the yaw rotation motion, which is helpful in avoiding the collisions. Six different cases were designed to test this system and the validity of the solutions was checked by using a non-linear model of the vehicles. In second research, Chakraborty et al. (2013) have analyzed that how forcefully time optimal maneuver performed by a cognitive vehicle can lessen the chances of the T-bone crash between two vehicles. It is stated that these maneuvers can be more efficient when the speed of the vehicle is high or friction of the road is low. The main difference is that the active front steering (AFS) is used to perform an optimal steering maneuver to avoid the T-bone collision. The drawback of research performed in Chakraborty et al. (2011, 2013) is that vehicles are acting like human drivers, without considering human behavior and emotions, to mitigate the effects of T-bone collisions. Furthermore, machine-learning capabilities, using supervised/unsupervised neural networks, can be introduced to the proper agent based model. It will help to perform more optimal collision mitigation maneuver.

Jeon et al. (2015) have proposed an autonomous emergency braking (AEB) technique using dedicated short range communication (DSRC) IEEE 802.11p based V2V communication to avoid intersection collisions. By evaluating different road conditions, the optimal time to apply brake has been determined. It has been verified that the performance of the conventional AEB system is affected at the speed of $60 \mathrm{~km} / \mathrm{h}$. The authors have also concluded that the conventional AEB system fails on snow and wet roads. Whereas the proposed AEB system has shown good results for avoiding collisions over snow, dry, and wet intersection roads. The drawback of this research work is same as mentioned by Joerer et al. (2014).

Table 5 presents summary of semi-autonomous car based intersection collision warning/avoidance systems.

g. Semi-autonomous car-agents inspired intersection/T-bone-collision detection/avoidance Traffic lights play an important role in intersection collision avoidance. However, there are many intersections, which have not the facility of traffic lights. To overcome this issue, Lee and Park (2012) have proposed the concept of intelligent agent-based virtual traffic lights over intersections to avoid the collisions. It provides cooperation between vehicles and infrastructures. The agent using projected trajectories has computed the intersection point of two vehicles. If there is a possibility of collision, then trajectories are adjusted. All vehicles are equipped with communication devices. The agent also provides the speed declaration guidelines to the vehicles to avoid a collision. Although, agent approach has been used in this research work. However, the proper agent architecture of proposed agent has not been presented. Furthermore, standard agent simulation software has not been used to develop and test the proposed technique.

Road traffic rules help the vehicles to regulate their movements without any conflict. However, there is a need of introducing such mechanism, unavailable for uncontrolled intersections, which help to communicate the vehicles with each other and resolve their conflicts by following the traffic rules. This safety issue for uncontrolled intersection has been addressed by Lu et al. (2014). The authors have proposed the set of rules, which help in identifying the sequence of the vehicle to pass through intersections. These rules 
Table 5 Overview of semi-autonomous car: intersection collision warning/avoidance systems

\begin{tabular}{|c|c|c|c|c|}
\hline S.no & Contribution & $\begin{array}{l}\text { Communication } \\
\text { method }\end{array}$ & Simulation/practical & References \\
\hline 1 & $\begin{array}{l}\text { An agent based collision } \\
\text { avoidance system over } \\
\text { signalized intersection is } \\
\text { proposed }\end{array}$ & $\begin{array}{l}\text { Vehicle-2-infrastructure } \\
\text { communication with } \\
\text { RSUs installed over } \\
\text { intersections }\end{array}$ & Simulation & Lee et al. (2013) \\
\hline 2 & $\begin{array}{l}\text { An intersection collision } \\
\text { avoidance protocol has } \\
\text { been proposed using } \\
\text { the collision probability } \\
\text { metric to compute the } \\
\text { chances of accidents }\end{array}$ & $\begin{array}{l}\text { Inter-vehicle communica- } \\
\text { tion uses IEEE } 802.11 p\end{array}$ & Simulation & Joerer et al. (2014) \\
\hline 3 & $\begin{array}{l}\text { The proposition of a } \\
\text { vehicle collision warning } \\
\text { system based on the } \\
\text { concept of internet- } \\
\text { connected Vehicles. }\end{array}$ & Internet cloud & Simulation & Liu et al. (2012) \\
\hline 4 & $\begin{array}{l}\text { The proposition of a } \\
\text { driver assistance System } \\
\text { (DAS) using the V2V } \\
\text { communication system } \\
\text { to avoid the intersection } \\
\text { collisions }\end{array}$ & $\begin{array}{l}\text { V2V using dedicated short } \\
\text { range communication } \\
\text { (DSRC) IEEE 802.11p }\end{array}$ & Practical & von Eichhorn et al. (2013) \\
\hline 5 & $\begin{array}{l}\text { An intersection collision } \\
\text { avoidance (ICA) system } \\
\text { is proposed using wire- } \\
\text { less sensor nodes (WSN) }\end{array}$ & $\begin{array}{l}\text { Vehicle-2-road (V2R) } \\
\text { communication using } \\
\text { Radiotronix Wi.232DTS a } \\
\text { low-power, embedded } \\
\text { radio transceiver oper- } \\
\text { ates on the 902-928 } \\
\text { MHz public frequency }\end{array}$ & Practical/simulation & Basma et al. (2011) \\
\hline 6 & $\begin{array}{l}\text { The proposition of an } \\
\text { intelligent V2I-based } \\
\text { urban area traffic man- } \\
\text { agement system and } \\
\text { evaluation of the traffic } \\
\text { situation using the fuzzy } \\
\text { traffic management } \\
\text { system }\end{array}$ & $\begin{array}{l}\text { Vehicle-2-infrastructure } \\
\text { communication using } \\
\text { wireless accesses for } \\
\text { vehicular environment } \\
\text { (WAVE) IEEE 802.11p }\end{array}$ & Simulation & Milanés et al. (2012c) \\
\hline 7 & $\begin{array}{l}\text { The proposition of a clus- } \\
\text { ter-based architecture } \\
\text { for intersection collision } \\
\text { avoidance using long } \\
\text { term evolution (LTE) and } \\
\text { Wi-Fi technologies }\end{array}$ & $\begin{array}{l}\text { Long term evolution (LTE) } \\
\text { and wireless fidelity } \\
\text { (Wi-Fi) }\end{array}$ & Simulation & Tung et al. (2013) \\
\hline 8 & $\begin{array}{l}\text { The proposition of an } \\
\text { intersection collision } \\
\text { avoidance technique } \\
\text { using autonomous } \\
\text { emergency braking } \\
\text { (AEB) technique and V2V } \\
\text { communication }\end{array}$ & $\begin{array}{l}\text { V2V using dedicated short } \\
\text { range communication } \\
\text { (DSRC) IEEE 802.11p }\end{array}$ & Simulation & Jeon et al. (2015) \\
\hline
\end{tabular}

are designed after the road traffic rules. Each approaching car makes decisions according to the rules based on exchanging information using vehicle-2-vehicle communication. The rule-based algorithm gives deceleration rate value to the vehicle when it encounters an approaching vehicle from the opposite direction. The car's braking system uses this value to avoid a collision. Although, traffic rules have been used to design this approach, social norms inspired agents might be explored for the same purpose. Simple rules based 
system can make some wrong decisions like "Do not give the way to the ambulance, if your priority is high". Social norms in combination with traffic rules might be useful to propose self-ethics based intersection CAS to overcome such issue. Furthermore, cognitive radio based $\mathrm{V} 2 \mathrm{~V}$ communication system can be used to enhance the performance of system in highly congested intersections.

Drivers make many decisions during driving, some of them are correct and some are incorrect. Incorrect safety decisions might cause severe road collisions. A mechanism having the capability to monitor the drivers' safety decisions and then correct them might be useful in avoiding collisions. In this regard, a mathematical model of the agent based driver assistant system has been presented by Colombo (2014) to avoid intersection collisions. The author has suggested a system that helps the vehicle to sense its position and share this information with another vehicle. In addition, a concept of an intelligent agent acting as supervisor installed within the vehicle has been introduced, which monitors the safety decisions taken by human drivers and corrects them if there is a chance of collision. The system has been tested successfully over intersections.

h. Semi-autonomous car-pedestrian collision detection/warning A vehicle-pedestrian CAS has been proposed by Nakagami et al. (2014). To avoid a collision, the authors focused on vehicle to pedestrian communication (VPEC), which applies inter-vehicle communication (IVC) technology. The authors develop pedestrian-vehicular collision avoidance support system (P-VCASS). By using a wireless LAN in P-VCASS, the information such as direction, velocity, and location are exchanged between vehicles and pedestrians. The proposed system consists of two algorithms: The first algorithm predicts the actions of a pedestrian using the moving record, whereas second one calculates the accumulation value of the degree of risk of a pedestrian. The validity of the proposed system was shown by experiments conducted in the Kansai University Takatsuki.

To avoid pedestrian-vehicle collision, a vision-based driver assistance system has been presented by Chien et al. (2013). It uses the fuzzy rule-based system to analyze driver's eyes and head motion. The proposed system detects the driver's line of sight, boundary detection, lane types, and the leading vehicle. The safety detection of pedestrians is computed using the gap between car and pedestrian. From the experiments, it was shown that in $93.18 \%$ of the test cases, pedestrians have been identified successfully in front of the vehicle.

Waizman et al. (2015) have presented a microscopic 3D, multi-agent simulation known as SAFEPED to avoid the pedestrian-vehicle collision at black spots. The SAFEPED is proficient of arbitrarily implementing cognitive-perceptual parameter of the pedestrian and the driver's behavior, which include tactical and strategic behavioral components. It is done by the assignment of human-based behavioral rules to the model agents. SAFEPED also helps to serve as a tool for the assessment of the risk of an accident at specific spots, and it can recognize safety measures to lower the risks. SAFEPED also serves as a tool for assessing the modifications to the existing and hypothetical black spots. In the future work of SAFEPED, more intricate behavior models of agents will be included. 
i. Semi-autonomous car-pedestrian collision avoidance A novel sliding force-based collision avoidance model has been introduced by Buisson et al. (2013). For the smooth collision avoidance, a new force is introduced known as sliding force. This force keeps the individual away from the obstacle and guides it to target's position. The forces in this model have used time-to-collision (TTC) parameter to avoid the collision from the obstacle. The obstacle's velocity is inherently integrated. This also ensures the smooth avoidance and greatly lessens the computational intricacy of the model. The proposed model has been effectively applied to avoid the collisions between bicyclists and pedestrians in a regular environment.

Edwards et al. (2015) have proposed the AEB system in vehicles to improve the pedestrian's safety. The major aim of this work was the safety of road users with the help of passive safety systems and AEB. This system activates the brakes one second earlier to the predicted impact with a pedestrian. The speed reduction in AEB is used to determine the speed at which pedestrian is impacted. Using Euro NCAP pedestrian impact or tests, injury probabilities are calculated. It was concluded that by fitting AEB system the rating of the passive safety system can be increased from poor to an average one.

j. Semi-autonomous car-animal collision detection/warning Zahrani et al. (2011) have developed a novel GPS based camel-vehicle accident avoidance system (CVAAS). GPS is used in this work to detect the direction, movement, and position of a camel. The system detects the presence of camel on or near the highway, and then a GPS sends the signal to the dedicated short range communication (DSRC) transmitter. The position of the camel is forwarded to DSRC receiver, which is mounted on the warning system. The warning system then warns the driver to avoid possible collision with the camel. Moreover, the danger zones are also classified in this system to adapt the alarming period.

Ewert (1996) have presented an animal collision avoidance system. It utilizes the electromagnetic transmissions for various purposes. It helps to alert the driver about unexpected collisions such as collision with animals, joggers, emergency vehicles, pedestrians, disabled vehicles, and bicyclists, etc. It also alerts people using the roadway to free the road. A speaker is fixed on the vehicle to send a message to the animal or people on the road to leave the path of approaching vehicles. The data about the dangerous position is received by the controller through a radio and is displayed to the driver for appropriate action.

Mammeri et al. (2014) have investigated a moose detection system to warn the driver about the danger when a large animal (i.e. Moose) is about to cross the roadway. The architecture of the system is designed according to two criteria: detection accuracy and recognition speed. To achieve these requirements, a two-stage approach system is investigated. In the first stage, a very fast LBP (powerful texture descriptor) AdaBoost algorithm is applied. This algorithm supplies the second stage by the RIOs, which contains the moose and other, related objects. In the second stage, because of good performance in classification and detection, HOG-SVM is used. To train and test the system, the authors created the data set. The system is tested over 1700 images and 10 videos. The system has been found efficient in these tests. 
k. Semi-autonomous car: cloud inspired road collision detection/warning In Abid et al. (2011) a new architecture of V-cloud is presented for the better operation of the VCPS by Abid et al. The proposed architecture is composed of three layers namely in car vehicular cyber-physical system (VCPS), vehicle-to-vehicle network (V2V) and vehicle-to-infrastructure network (V2I) layers. Two types of different sensors are used in the in-car layer: Vehicle's internal sensors and smartphone sensors. The in-car system helps in tracking the driver behavior. V2V and V2I communication are carried out using DSRC, Wi-Fi, or 3G 4 G networks in order to avoid the rear end collisions. The in-car sensors ignore the emotional aspects while tracking human behavior. Further, DSRC is used, which is proved inefficient to handle the bandwidth demand of highly crowded highways.

In Wan et al. (2014b), a multi-layer context-aware architecture using cloud support has been proposed for VCPS. The three layers of this architecture are a vehicle, location, and cloud. The authors have proposed the solution to solve the context-aware safety hazard prediction using the concept of field theory. According to the authors, the specific shape of the potential field depends on driver characteristics as well as context-aware information. V2V communication is used for the availability of context-aware information. However, the authors do not consider human emotions as one of the basic characteristics of the driver. Furthermore, single radio access technology (RAT) is used for exchanging context-aware information. According to Riaz et al. (2015b) single RAT can build incomplete field potential and hence, rear end collision algorithms cannot performed at their best. This is the drawback of this research work and it can be improved using multi-RAT concept proposed by Riaz et al. (2015b).

Mobile cloud computing (MCC) supported VCPS architecture is proposed by Wan et al. (2014a). The proposed architecture is composed of four layers. The first layer is a microlayer, which emphasizes on two important aspects. The first aspect is the designing, evaluating human factors based applications for improved traffic safety and actions. The second aspect is designing the traffic aware mobile GIS. In the second layer, the safety information and entertainment resources are shared with the drivers or passengers. The third layer macro is responsible for the communication between users cloud services. The two important components of cloud-supported services are geographic information system (GIS) with traffic-aware capability and cloud-supported dynamic vehicle routing. The missing part of research is ignoring the human emotions while designing road safety applications by evaluating human factors.

l. Role of emotions in enhancing performance of semi-autonomous VCPS Emotions have proven contribution in building humans' mental stress, and this stress can be released by expressing them properly. In real social life, humans can express their emotions by communicating with each other. However, drivers have not such a mechanism, while driving to release their mental stress, to express their emotions. To overcome this issue, Kim and Lee (2015) have proposed an emotions expressing system, Which enable the drivers to express their emotions to the other drivers. The proposed system helps the drivers in realizing their stress and hence they drive more safely. The authors claimed that the emotions like anger, apology, and gratitude could not be easily communicated between drivers. To understand the context of the driving and emotions, in different situations, seven participants were recruited. Furthermore, the prototype consists of Arduino microcontroller 
and a dot matrix display was built to show the drivers emotions. For the experiment, this prototype was installed in the car environment and validated through an experiment conducted on seven novice drivers. Although, the authors have proposed a mechanism for the drivers to express their emotions, they have not studied the effects of emotions expressed by a driver in increasing the mental stress of another driver. For example, if a driver is feeling anger and he/she shows the icon of anger to the neighboring driver, it might make him/her angry and he/she starts rash driving. Furthermore, it would be interesting to study the same concept for the autonomous vehicles, which exchange their emotions using machine-to-machine (M-2-M) communication. In this way autonomous cars will be able to update each other about critical situations using different emotions and the collisions might be handled more robustly.

Humans are cognitive as well emotional in nature and prefer those companions which understand their emotions and help them to cope with different tragedies of life. The same companion is required for human drivers, which help them to handle different emergency situations by adapting itself according to their emotions. In this regard, Reichardt (2008) has presented emotions inspired driver's assistant model which simulates the emotional influence on the human driver's behavior. The main purpose of this model is to build a framework for learning algorithms, which will be used in the adaptive driver assistance system. The cognitive appraisal model is used by integrating it with a model of risk. Further cognitive appraisal model of emotion is integrated with situation assessment for the efficient assistance of a driver. The main contribution is an artificial emotional agent, which has the ability to show adequate emotion according to different situations. The missing part of research is that authors have proposed an emotional agent without presenting proper emotion generation mechanism. Furthermore, the authors have simulated the so called emotional agent using non standard agent simulation. In addition, results are not valid as well. Emotions are fuzzy and qualitative in nature. It would be interesting to compute the emotions quantitatively and for this purpose fuzzy logic might be explored.

Affective computing is the field, which helps in computing the emotions using different methods. The question is such driver assistance systems can be devised, which use affective computing to compute the current emotional state of drivers and help them to avoid the collisions. To answer this question, Lisetti and Nasoz (2005) have proposed an affective computing inspired intelligent car interface by facilitating a natural human interaction with drivers. For this purpose, they map different physiological signals like a heartbeat, temperature, and response to the driving related emotions and states. A driving experiment was designed and conducted in a virtual reality environment. The Physiological signals were analyzed using different algorithms like KNN, MBP, and Resilient back propagation. The results showed that KNN classifies these emotions with $66.3 \%$, MBP with $76.7 \%$ and RBP classify them with $91.9 \%$ accuracy.

Driver assistance systems (DAS), having capability to recognize driver emotions along with facial gestures, can assist the drivers in a better way. In this regard, many research studies have been performed to recognize human emotions and facial gestures. However, simultaneous emotion recognition and facial gesture tracking is a challenging task. To overcome this issue, a fuzzy inference system is presented to perform emotion recognition and facial gesture tracking at a same time by Agrawal et al. (2013). It has 
been analyzed by the authors that sometimes it is not possible for the drowsy and tired driver to take advantage of DAS. Simultaneous facial expression detection and emotion inference is necessary for encouraging automation in DAS. For this purpose, a complete model has been described and tested for detection of facial expressions and emotions. Face detection has been performed using classifier and centroid calculations. Emotions were analyzed using fuzzy bases inference system. However, the authors have proposed an emotion recognition model without considering a proper emotion appraisal model.

Table 6 presents summary of the role of emotions in enhancing performance of semiautonomous VCPS.

\section{Open research problems}

In this section, open research problems are discussed to help the researchers working in VCPS related field.

\section{Lack of human inspired design}

Most of the above discussed VCPS are assisting humans without having the humans inspired design, which indicates an obvious cooperation gap between both current VCPS and drivers. Hence, our key problem with existing VCPS is that while humans are extremely emotional in their decision-making, existing VCPS have been not designed with human emotions in mind and, therefore, it is a poor match. In the design of existing VCPS, the role of affective computing has not been explored. Research studies by Chakraborty et al. (2011, 2013), Abid et al. (2011), Li et al. (2014a, b), Czubenko et al. (2015), Llorca et al. (2011), An et al. (2014), Chang and Chou (2009), Keller et al. (2014), Milanés et al. (2012a), Kim and Jeong (2014), Wan et al. (2014a) and Kraus et al. (2009)

Table 6 Overview of role of emotions in enhancing performance of semi-autonomous vcps

\begin{tabular}{|c|c|c|c|c|}
\hline S.no & Contribution & Emotions used & Simulation/practical & References \\
\hline 1 & $\begin{array}{l}\text { The proposition of an emo- } \\
\text { tions expressing system } \\
\text { which helps the drivers } \\
\text { to express their emotions } \\
\text { to the other drivers }\end{array}$ & $\begin{array}{l}\text { Anger, apology, and } \\
\text { gratitude }\end{array}$ & Practical & Kim and Lee (2015) \\
\hline 2 & $\begin{array}{l}\text { Orthony, clore, and collins } \\
(\text { OCC) inspired driver's } \\
\text { assistant model which } \\
\text { simulates the emotional } \\
\text { influence on the human } \\
\text { driver's behaviour }\end{array}$ & Fear, anger, and gratitude & Simulation & Reichardt (2008) \\
\hline 3 & $\begin{array}{l}\text { The proposition of an } \\
\text { intelligent car interface } \\
\text { by facilitating a natural } \\
\text { human interaction with } \\
\text { the drivers so that he/ } \\
\text { she will be aware of his } \\
\text { emotional state during } \\
\text { driving }\end{array}$ & $\begin{array}{l}\text { Sadness, anger, surprise, } \\
\text { fear, frustration, and } \\
\text { amusement }\end{array}$ & Practical & Lisetti and Nasoz (2005) \\
\hline 4 & $\begin{array}{l}\text { A fuzzy inference system is } \\
\text { presented for improving } \\
\text { the performance of the } \\
\text { emotions inspired driver } \\
\text { assistance system (DAS) }\end{array}$ & $\begin{array}{l}\text { Happiness, anger, sad, and } \\
\text { surprise }\end{array}$ & Practical & Agrawal et al. (2013) \\
\hline
\end{tabular}


have not considered emotions in the design of collision avoidance schemes. It would be interesting to explore affective computing to design more human compatible VCPS to tailor more efficient collision avoidance schemes.

\section{Lack of efficient communication protocol}

The main drawback of the proposed intersection collision avoidance techniques in Abid et al. (2011), Xiang et al. (2014), Joerer et al. (2014), von Eichhorn et al. (2013), Milanés et al. (2012c) and Tung et al. (2013) is that they are using IEEE 802.11p for IVC purpose. IEEE $802.11 \mathrm{p}$ protocol is using seven $10 \mathrm{MHz}$ wide channels in the range of $5.9 \mathrm{GHz}$ spectrum. However, it has been found that these dedicated frequency resources fail to solve the problem of bandwidth allocation due to the increasing number of users (vehicles) competing for same channel within the same area (Riaz et al. 2015a). Furthermore, the delay in safety messages should be less than $200 \mathrm{~ms}$ in vehicular adhoc networks (VANETs). Whereas, due to data contention in the control channel of DSRC the safety message delivery time, packets have to be resent many times, exceeds $1000 \mathrm{~ms}$. It means the proposed intersection collision avoidance schemes will not work properly on the highly congested intersection scenario. It would be interesting to evaluate cognitive radio based solutions to overcome this issue.

\section{Lack of proper agent based modeling}

The missing part of research in Benine-Neto et al. (2014), Lee et al. (2013), Lee and Park (2012) and Colombo (2014) is that proper agent based modeling paradigm has not been followed to design the agent based collision avoidance systems. Furthermore, the proper agent architecture has not been proposed. In addition, non-standard agent simulation software is used for the development and testing of the proposed technique. It would be interesting to use proper agent based modeling (ABM) in the design of these collision avoidance schemes along the standard agent based simulation environment like Netlogo or Starlogo.

\section{Modeling VCPS as complex adaptive system}

The operation of VCPS comprises traffic entities along with environmental and animal/ pedestrian entities. The existing literature lacks such models, which consider all of these stakeholders in a single model. In literature, agent-based modeling (ABM) is utilized to model communication networks considering the environment, animals, and pedestrian. However, any model in the context of high-speed VCPS along with the environment and other entities has not been presented yet. Niazi (2013) has reported ABM as a potential candidate for modeling complex adaptive system like wireless sensor networks, swarm robotic networks, self-assembling robots, peer-to-peer networks. Niazi and Hussain (2009) have presented a self-organizable agent-based model for P2P/ad hoc networks and complex systems keeping in mind their interaction with environment/humans/ animals. Niazi and Hussain (2011a) have presented a novel formal agent-based simulation framework (FABS) to improve the sensing capability of wireless sensor networks in a complex adaptive environment. In addition, Niazi and Hussain (2011b) have proposed sensing of emergent behavior in a complex adaptive system (SECAS) using ABM. In Batool and Niazi (2015), an agent-based model has been proposed for self-organized 
power consumption approximation in the internet of things. Modeling of the IOT has also been discussed by Laghari and Niazi (2016). The existing literature uses ABM in the context of complex adaptive system. Cyber-physical systems have not proven the type of CAS. It would be interesting to explore ABM for non-CAS systems like VCPS.

\title{
Conclusions
}

Road accidents are caused due to numerous reasons. The goal of the current review is to give an extensive review of literature related to collision avoidance solutions primarily with a focus on the aspects of communication in the domain of vehicular cyber-physical systems. We suggest the use of emotions and affective computing as well as a novel taxonomy for understanding VCPS concepts. The idea is to assist researchers in locating key references for existing collision avoidance solutions. We have highlighted how neglecting the cognitive state of drivers in autonomous and semi-autonomous vehicles can severely affect the design of future VCPS systems. We believe that the presented review will expand the horizons of understanding in the domain of VCPS.

\begin{abstract}
Abbreviations
ACC: adaptive cruise control systems (Reddy 2015); AV: autonomous vehicle (Iftekhar and Olfati-Saber 2012); AEB: autonomous emergency braking (Jeon et al. 2015); CABC: cognitive agent-based computing (Niazi and Hussain 2012); CAS: complex adaptive system (Niazi and Hussain 2011a); CPS: cyber-physical systems (Poovendran 2010); CAS: collision avoidance system (Milanés et al. 2012C); CWS: collision warning system (Milanés et al. 2012C); DSRC: dedicated short range communication (Xiang et al. 2014); ECU: electronic control unit (Xiang et al. 2014); ICA: intersection collision avoidance (Basma et al. 2011); LTE: long term evolution (Tung et al. 2013); LDA: linear discrimination analysis (An et al. 2014); LDW: lane departure warning (Kusano and Gabler 2012); OSS: on-board safety systems (Jeffrey et al. 2015); RDP: road departure prevention (Katzourakis et al. 2014); MCC: mobile cloud computing (An et al. 2014); VCPS: vehicular cyberphysical systems (Reddy 2015); V2V: vehicle-2-vehicle (Abid et al. 2011); V2I: vehicle-2-infrastructure (Milanés et al. 2012C); WSN: wireless sensor networks (Basma et al. 2011; Sohrabi et al. 2000); XAV: experimental autonomous vehicle (Park 2008); WAVE: wireless accesses for vehicular environment (Milanés et al. 2012c).
\end{abstract}

\section{Authors' contributions}

FR and MN have equally contributed to the paper in both drafting the manuscript as well as revising it. Both authors read and approved the final manuscript.

\section{Author details}

${ }^{1}$ Department of Computing-Iqra University, Islamabad, Pakistan. ${ }^{2}$ Department of Computer Sciences-COMSATS, Islamabad, Pakistan.

\section{Acknowledgements}

We are thankful to MUST and COMSATS for facilitating us in this project.

\section{Competing interests}

The authors declare that they have no competing interests.

Received: 19 December 2015 Accepted: 6 July 2016

Published online: 22 July 2016

\section{References}

Abid H, Phuong LTT, Wang J, Lee S, Qaisar S (eds) (2011) V-Cloud: vehicular cyber-physical systems and cloud computing. In: Proceedings of the 4th international symposium on applied sciences in biomedical and communication technologies. ACM, New York

Administration NHTS (2013) Preliminary statement of policy concerning automated vehicles. Washington, DC

Agrawal U, Giripunje S, Bajaj P (eds) (2013) Emotion and gesture recognition with soft computing tool for drivers assistance system in human centered transportation. In: IEEE international conference on systems, man, and cybernetics (SMC). IEEE, New York

Al-Zaher TSA, Bayoumy AM, Sharaf A, El-din YHH (eds) (2012) Lane tracking and obstacle avoidance for autonomous ground vehicles. In: Mechatronics (MECATRONICS). In: 13th Int'I Workshop on 9th France-Japan \& 7th Europe-Asia Congress on and research and education in mechatronics (REM). IEEE, New York

An J, Choi B, Kim B, Kim E, Hwang J (eds) (2014) Rear-end collision warning system using linear discriminant analysis. In: 15th international symposium on soft computing and intelligent systems (SCIS), Joint 7th international conference on and advanced intelligent systems (ISIS). IEEE, New York

Anderson JM, Nidhi K, Stanley KD, Sorensen P, Samaras C, Oluwatola OA (2014) Autonomous vehicle technology: a guide for policymakers. Rand Corporation, Santa Monica 
Anderson SJ, Peters SC, lagnemma KD, Pilutti TE (eds) (2009) A unified approach to semi-autonomous control of passenger vehicles in hazard avoidance scenarios. In: IEEE international conference on systems, man and cybernetics SMC. IEEE, New York

Baheti R, Gill H (2011) Cyber-physical systems. Impact Control Technol 12:161-166

Basma F, Tachwali Y, Refai HH (eds) (2011) Intersection collision avoidance system using infrastructure communication. In: 14th international IEEE conference on intelligent transportation systems (ITSC). IEEE, New York

Batool K, Niazi MA (eds) (2015) Self-organized power consumption approximation in the internet of things. In: IEEE international conference on consumer electronics (ICCE). IEEE, New York

Benavente M, Rothenberg HA, Knodler Jr MA (eds) (2006) Evaluation of frequency and injury outcomes of lane departure crashes. In: 2006 ITE annual meeting and exhibit compendium of technical Papers

Bengler K, Dietmayer K, Farber B, Maurer M, Stiller C, Winner H (2014) Three decades of driver assistance systems: review and future perspectives. Intell Transp Syst Mag IEEE 6(4):6-22

Benine-Neto A, Scalzi S, Mammar S, Netto M, Lusetti B (2014) Model reference-based vehicle lateral control for lane departure avoidance. Int J Veh Auton Syst 12(3):284-306

Bennet SA, Yiannakoulias N (2015) Motor-vehicle collisions involving child pedestrians at intersection and mid-block locations. Accid Anal Prev 78:94-103

Bimbraw K (ed) (2015) Autonomous cars: past, present and future a review of the developments in the last century, the present scenario and the expected future of autonomous vehicle technology. In: 12th international conference on informatics in control, automation and robotics (ICINCO). IEEE, New York

Broggi A, Bertozzi M, Fascioli A, Bianco CGL, Piazzi A (1999) The ARGO autonomous vehicle's vision and control systems. Int J Intell Control Syst 3(4):409-441

Buisson J, Galland S, Gaud N, Gonçalves M, Koukam A (2013) Real-time collision avoidance for pedestrian and bicyclist simulation: a smooth and predictive approach. Procedia Comput Sci 19:815-820

Cabrera A, Gowal S, Martinoli A (eds) (2012) A new collision warning system for lead vehicles in rear-end collisions. In: 2012 IEEE intelligent vehicles symposium (IV). IEEE, New York

Carod-Artal F (2016) Epidemiology and neurological complications of infection by the Zika virus: a new emerging neurotropic virus. Revista de neurologia 62(7):317

Chakraborty I, Tsiotras P, Diaz RS (eds) (2013) Time-optimal vehicle posture control to mitigate unavoidable collisions using conventional control inputs. In: 2013 American control conference. IEEE, New York

Chakraborty I, Tsiotras P, Lu J (eds) (2011) Vehicle posture control through aggressive maneuvering for mitigation of T-bone collisions. In: 50th IEEE conference on decision and control and European control conference. IEEE, New York

Chan M, Singhal A (2013) The emotional side of cognitive distraction: implications for road safety. Accid Anal Prev 50:147-154

Chang C-Y, Chou Y-R (2009) Development of fuzzy-based bus rear-end collision warning thresholds using a driving simulator. IEEE Trans Intell Transp Syst 10(2):360-365

Chen C, Zhang G, Tarefder R, Ma J, Wei H, Guan H (2015) A multinomial logit model-Bayesian network hybrid approach for driver injury severity analyses in rear-end crashes. Accid Anal Prev 80:76-88

Chen Y, Sun Y, Ding N, Chung WK, Qian H, Xu G et al (eds) (2012) A real-time vehicle safety system. In: 2012 IEEE/SICE international symposium on system integration (SII). IEEE, New York

Chien J-C, Lee J-D, Chen C-M, Fan M-W, Chen Y-H, Liu L-C (2013) An integrated driver warning system for driver and pedestrian safety. Appl Soft Comput 13(11):4413-4427

Chun J, Lee I, Park G, Seo J, Choi S, Han SH (2013) Efficacy of haptic blind spot warnings applied through a steering wheel or a seatbelt. Transp Res Part Traffic Psychol Behav 21:231-241

Colombo A (ed) (2014) A mathematical framework for cooperative collision avoidance of human-driven vehicles at intersections. In: 11th international symposium on wireless communications systems (ISWCS). IEEE, New York

Cord M (2009) An approach to simula vehicles in intense. Universidade do Porto

Costanzo A, Faro A, Giordano D, Pino C (eds) (2016) Mobile cyber physical systems for health care: Functions, ambient ontology and e-diagnostics. In: 13th IEEE Annual consumer communications \& networking conference (CCNC). IEEE, New York

Crocetta G, Piantini S, Pierini M, Simms C (2015) The influence of vehicle front-end design on pedestrian ground impact. Accid Anal Prev 79:56-69

Czubenko M, Kowalczuk Z, Ordys A (2015) Autonomous driver based on an intelligent system of decision-making. Cognit Comput 7:1-13

Dabbour E, Easa S (2014) Proposed collision warning system for right-turning vehicles at two-way stop-controlled rural intersections. Transp Res Emerg Technol 42:121-131

Dagale H, Anand S, Hegde M, Purohit N, Supreeth M, Gill GS et al (eds) (2015) CyPhyS+: a reliable and managed cyberphysical system for old-age home healthcare over a 6 LoWPAN using wearable motes. In: IEEE international conference on services computing (SCC). IEEE, New York

Daza IG, Bergasa LM, Bronte S, Yebes JJ, Almazán J, Arroyo R (2014) Fusion of optimized indicators from advanced driver assistance systems (ADAS) for driver drowsiness detection. Sensors 14(1):1106-1131

Dickmanns ED, Behringer R, Dickmanns D, Hildebrandt T, Maurer M, Thomanek F et al (eds) (1994) The seeing passenger car'VaMoRs-P'. In: Proceedings of the intelligent vehicles' 94 symposium. IEEE, New York

Doniec A, Mandiau R, Piechowiak S, Espié S (2008) A behavioral multi-agent model for road traffic simulation. Eng Appl Artif Intell 21(8):1443-1454

Edwards MNA, Carroll J, Wisch M, Zander O, Lubbe N (2015) Assessment of integrated pedestrian protection systems with autonomous emergency braking (AEB) and passive safety components. Traffic Inj Prev 16:2-11. doi:10.1080/1538 9588.2014.1003154

Ewert RD (1996) Animal collision avoidance system for a vehicle. Google Patents

Fink DA (2014) The prevention of road accidents in the countries of Europe, Asia and the United States of America. World Appl Sci J 30(12):1863-1869 
Fleming W (2015) Forty-year review of automotive electronics: a unique source of historical information on automotive electronics. Veh Technol Mag IEEE 10(3):80-90

Funke J, Theodosis P, Hindiyeh R, Stanek G, Kritatakirana K, Gerdes C et al (eds) (2012) Up to the limits: autonomous Audi TTS. In: IEEE intelligent vehicles symposium (IV). IEEE, New York

Goodrich J (2013) Driving miss daisy: an autonomous chauffeur system. Hous L Rev 51:265

Gray A, Ali M, Gao Y, Hedrick JK, Borrelli F (eds) (2012) Integrated threat assessment and control design for roadway departure avoidance. In: 15th international IEEE conference on intelligent transportation systems (ITSC). IEEE, New York

Gulzar S YF, Mir Z, Zafar R (2012) Provincial analysis of traffic accidents in Pakistan

(2015) Highway statistics 2013. Washington, DC: US Department of Transportation

Harb R, Radwan E, Yan X, Abdel-Aty M (2007) Light truck vehicles (LTVs) contribution to rear-end collisions. Accid Anal Prev 39(5):1026-1036

Hilhorst D (2002) Being good at doing good? Quality and accountability of humanitarian NGOs. Disasters 26(3):193-212

Huang KC, Huang TY, Chuang CH, King JT, Wang YK, Lin CT et al (2016) An EEG-based fatigue detection and mitigation system. Int J Neural Syst 26:1650018

Huang Z, Wu Y, Liu J (2015) Research on effects of pattern, amplitude and frequency of pulse steering torque warnings for lane departure. Transp Res Traffic Psychol Behav 31:67-76

Iftekhar L, Olfati-Saber R (eds) (2012) Autonomous driving for vehicular networks with nonlinear dynamics. In: 2012 IEEE intelligent vehicles symposium (IV). IEEE, New York

Jeffrey S, Hickman FG, Camden MC, Richard J, Hanowski RJ, Alejandra J, Erin M (2015) Efficiency of roll stability control and lane departure warning system using carrier collected data. J Safety Res 52:59-63

Jeon S, Kim G, Kim B (2015) Study on V2V-based AEB system performance analysis in various road conditions at an intersection. System 7:10

Joerer S, Segata M, Bloessl B, Locigno R, Sommer C, Dressler F (2014) A vehicular networking perspective on estimating vehicle collision probability at intersections. IEEE Trans Veh Technol 63(4):1802-1812

Kala R, Warwick K (2013) Planning autonomous vehicles in the absence of speed lanes using an elastic strip. IEEE Trans Intell Transp Syst 14(4):1743-1752

Katzourakis D, de Winter JC, Alirezaei M, Corno M, Happee R (2014) Road-departure prevention in an emergency obstacle avoidance situation. IEEE Trans Syst Man Cybern 44(5):621-629

Keller M, Hass C, Seewald A, Bertram T (eds) (2014) Driving simulator study on an emergency steering assist. In: IEEE international conference on systems, man and cybernetics (SMC). IEEE, New York

Kim H, Lee KP (eds) (2015) Express driver's emotion with emoticons in driving contexts. In: Proceedings of the 33rd annual ACM conference extended abstracts on human factors in computing systems. ACM, New York

Kim T, Jeong H-Y (2014) A novel algorithm for crash detection under general road scenes using crash probabilities and an interactive multiple model particle filter. IEEE Trans Intell Transp Syst 15(6):2480-2490

King JD (2004) Polarametric blind spot detector with steerable beam. Google Patents

Kok J, Gonzalez LF, Kelson N (2013) FPGA implementation of an evolutionary algorithm for autonomous unmanned aerial vehicle on-board path planning. IEEE Trans Evol Comput 17(2):272-281

Koopmans JM, Friedman L, Kwon S, Sheehan K (2015) Urban crash-related child pedestrian injury incidence and characteristics associated with injury severity. Accid Anal Prev 77:127-136

Kraus S, Althoff M, Heißing B, Buss M (eds) (2009) IEEE cognition and emotion in autonomous cars. Intelligent vehicles symposium. IEEE, New York

Kusano KD, Gabler HC (eds) (2012) Model of collision avoidance with lane departure warning in real-world departure collisions with fixed roadside objects. In: 2012 15th international IEEE conference on intelligent transportation systems. IEEE, New York

Laghari S, Niazi MA (2016) Modeling the internet of things, self-organizing and other complex adaptive communication networks: a cognitive agent-based Ccmputing approach. PloS One 11(1):e0146760

Langbein J (2007) National deer-vehicle collisions project: England 2003-2005. Final report to the highways agency the deer initiative, Wrexham

Lansdown TC, Stephens AN, Walker GH (2015) Multiple driver distractions: a systemic transport problem. Accid Anal Prev 74:360-367

Lari A, Douma F, Onyiah I (2014) Self-driving vehicles: current status of autonomous vehicle development and minnesota policy implications. University of Minnesota White Paper

Lee E-Y, Cho H-J, Lee S-W, Ryu K-Y (2013) Agent-based safety support system at intersections. Res Notes Inform Sci 14(48):279-282

Lee EA (ed) (2008) Cyber physical systems: design challenges. In: 11th IEEE international symposium on object oriented real-time distributed computing (ISORC). IEEE, New York

Lee I, Sha L, Stankovic J (2010) Cyber-Physical Systems: the next computing revolution. Adream, LAAS-CNSR-2010

Lee J, Park B (2012) Development and evaluation of a cooperative vehicle intersection control algorithm under the connected vehicles environment. IEEE Trans Intell Transp Syst 13(1):81-90

Li X, Qiao C, Yu X, Wagh A, Sudhaakar R, Addepalli S (2012) Toward effective service scheduling for human drivers in vehicular cyber-physical systems. IEEE Trans Parallel Distrib Syst 23(9):1775-1789

Li L, Lu G, Wang Y, Tian D (eds) (2014) A rear-end collision avoidance system of connected vehicles. In: 17th international IEEE conference on intelligent transportation systems (ITSC). IEEE, New York

Li X, Shaobin W, Li F (eds) (2014) Fuzzy based collision avoidance control strategy considering crisis index in low speed urban area. In: IEEE conference and expo transportation electrification asia-pacific (ITEC Asia-Pacific). IEEE, New York

Lisetti CL, Nasoz F (eds) (2005) Affective intelligent car interfaces with emotion recognition. In: Proceedings of 11th international conference on Human computer interaction, Las Vegas, Citeseer

Litman T (2014) In: Autonomous vehicle implementation predictions. Victoria transport policy institute 28 
Liu T, Wang Y, Wenjuan E, Tian D, Yu G, Wang J (eds) (2012) Vehicle collision warning system and algorithm at intersection under internet-connected vehicles environment. In: Proceedings of the 12th COTA international conference of transportation professionals: multimodal transportation systems (CICTP'12)

Llorca DF, Milanés V, Alonso IP, Gavilán M, Daza IG, Pérez J et al (2011) Autonomous pedestrian collision avoidance using a fuzzy steering controller. IEEE Trans Intell Transp Syst 12(2):390-401

Loss SR, Will T, Marra PP (2014) Estimation of bird-vehicle collision mortality on US roads. J Wildl Manag 78(5):763-771

Lu G, Li L, Wang Y, Zhang R, Bao Z, Chen H (eds) (2014) A rule based control algorithm of connected vehicles in uncontrolled intersection. In: IEEE 17th international conference on intelligent transportation systems (ITSC). IEEE, New York

Lukic SM, Cao J, Bansal RC, Rodriguez F, Emadi A (2008) Energy storage systems for automotive applications. IEEE Trans Ind Electron 55(6):2258-2267

Mammeri A, Zhou D, Boukerche A, Almulla M (eds) (2014) An efficient animal detection system for smart cars using cascaded classifiers. In: 2014 IEEE international conference on communications. IEEE, New York

Manawadu U, Ishikawa M, Kamezaki M, Sugano S (eds) (2015) Analysis of individual driving experience in autonomous and human-driven vehicles using a driving simulator. In: 2015 IEEE international conference on advanced intelligent mechatronics (AIM). IEEE, New York

Manley E (2014) Modelling driver behaviour to predict urban road traffic dynamics: UCL (University College London)

Markoff J (2010) Smarter than you think-google cars drive themselves, in traffic. NY Times article. http://www.nytimes. com/2010/10/10/science/10google.html

Meinel HH (ed) (2014) Evolving automotive radar-from the very beginnings into the future. In: 2014 8th European conference on antennas and propagation (EuCAP). IEEE, New York

Mersky AC, Samaras C (2016) Fuel economy testing of autonomous vehicles. Transp Res Emerg Technol 65:31-48

Milanés V, Llorca DF, Villagrá J, Pérez J, Fernández C, Parra I et al (2012a) Intelligent automatic overtaking system using vision for vehicle detection. Expert Syst Appl 39(3):3362-3373

Milanés V, Pérez J, Godoy J, Onieva E (2012b) A fuzzy aid rear-end collision warning/avoidance system. Expert Syst Appl 39(10):9097-9107

Milanés V, Villagrá J, Godoy J, Simó J, Pérez J, Onieva E (2012c) An intelligent V2I-based traffic management system. IEEE Trans Intell Transp Syst 13(1):49-58

Mon Y-J, Lin C-M (2012) Supervisory recurrent fuzzy neural network control for vehicle collision avoidance system design. Neural Comput Appl 21(8):2163-2169

Motwani A, Sharma S, Sutton R, Culverhouse P (2013) Interval Kalman filtering in navigation system design for an uninhabited surface vehicle. J Navig 66(05):639-652

Mutz F, Veronese LP, Oliveira-Santos T, de Aguiar E, Cheein FAA, De Souza AF (2016) Large-scale mapping in complex field scenarios using an autonomous car. Expert Syst Appl 46:439-462

Nakagami G, Yoshida T, Matsuoka M, Wada T, Okada H (eds) (2014) A new collision avoidance support system between pedestrian and vehicle using pedestrian moving record. In: 2014 IEEE 3rd global conference on consumer electronics (GCCE). IEEE, New York

Niazi MA (2013) Complex adaptive systems modeling: a multidisciplinary roadmap. Complex Adap Syst Model 1(1):1-14

Niazi M, Hussain A (2009) Agent-based tools for modeling and simulation of self-organization in peer-to-peer, ad hoc, and other complex networks. IEEE Commun Mag 47(3):166-173

Niazi M, Hussain A (2011a) A novel agent-based simulation framework for sensing in complex adaptive environments. IEEE Sens J 11(2):404-412

Niazi M, Hussain A (2011b) Sensing emergence in complex systems. IEEE Sens J 11(10):2479-2480

Niazi M, Hussain A (2012) Cognitive agent-based computing-l: a unified framework for modeling complex adaptive systems using agent-based \& complex network-based methods. Springer Science \& Business Media, 2012

Nishimura N, Simms CK, Wood DP (2015) Impact characteristics of a vehicle population in low speed front to rear collisions. Accid Anal Prev 79:1-12

Park TCJWHJSCY-W (2008) Real-time collision avoidance method for unmanned ground vehicle. In: International conference on control, automation and systems. ICCAS. p. 843-846

Patton GC, Coffey C, Sawyer SM, Viner RM, Haller DM, Bose K et al (2009) Global patterns of mortality in young people: a systematic analysis of population health data. Lancet 374(9693):881-892

Payre W, Cestac J, Delhomme P (2014) Intention to use a fully automated car: Attitudes and a priori acceptability. Transp Res Traffic Psychol Behav 27:252-263

Poovendran R (2010) Cyber-physical systems: close encounters between two parallel worlds. Proc IEEE 98(8):1363-1366

Poplin GS, McMurry TL, Forman JL, Hartka T, Park G, Shaw G et al (2015) Nature and etiology of hollow-organ abdominal injuries in frontal crashes. Accid Anal Prev 78:51-57

Rane A, Krishnan S, Waman S (eds) (2014) Conflict resolution of autonomous cars using game theory and cellular automata. In: 2014 international conference on optimization, reliabilty, and information technology (ICROIT). IEEE, New York

Rauskolb FW, Berger K, Lipski C, Magnor M, Cornelsen K, Effertz J et al (2008) Caroline: an autonomously driving vehicle for urban environments. J Field Robot 25(9):674-724

Reddy YB (ed) (2015) Security and design challenges in cyber-physical systems. In: 12th international conference on information technology-new generations (ITNG). IEEE, New York

Reichardt DM (ed) (2008) Approaching driver models which integrate models of emotion and risk. Intelligent vehicles symposium. IEEE, New York

Riaz F, Niazi MA, Sajid M, Amin S, Ratyal NI, Butt F (2015a) An efficient collision avoidance scheme for autonomous vehicles using genetic algorithm. J Appl Environ Biol Sci 5(8):70-76

Riaz F, Shafi I, Jabbar S, Khalid S, Rho S (2015b) A novel white space optimization scheme using memory enabled genetic algorithm in cognitive vehicular communication. Wireless Pers Commun 1-23

Riaz F, Shah SI, Raees M, Shafi I, Iqbal A (2013) Lateral pre-crash sensing and avoidance in emotion enabled cognitive agent based vehicle-2-vehicle communication system. Int J Commun Netw Inform Secur 5:2 
Rosey F, Auberlet J-M, Bertrand J, Plainchault P (2008) Impact of perceptual treatments on lateral control during driving on crest vertical curves: a driving simulator study. Accid Anal Prev 40(4):1513-1523

Rowden P, Steinhardt D, Sheehan M (2008) Road crashes involving animals in Australia. Accid Anal Prev 40(6):1865-1871 Rumschlag G, Palumbo T, Martin A, Head D, George R, Commissaris RL (2015) The effects of texting on driving performance in a driving simulator: the influence of driver age. Accid Anal Prev 74:145-149

Sailan K, Kuhnert K-D (2015) Speed control of unmanned ground vehicle for non autonomous operation. Int J Mater Sci Eng 3(1):44-49

Schittenhelm H (ed) (2013) Advanced brake assist-real world effectiveness of current implementations and next generation enlargements by Mercedes-Benz. In: Proceedings of the 23rd international technical conference on the enhanced safety of vehicles (ESV). Seoul

Schwindt O, Kim J, Chakraborty B, Buckner K, Ignaczak B (2015) In: Enhanced lane departure system. US Patent $20,150,183,430$

Shasthri S, Kausalyah V, Shah QH, Abdullah KA, Idres M, Wong SV (2015) Parametric study for head injury criteria response of three-year olds in a child restraint system in oblique and lateral intrusive side impact. Int J Crashworthiness 20(1):1-11

Sohrabi K, Gao J, Ailawadhi V, Pottie GJ (2000) Protocols for self-organization of a wireless sensor network. IEEE Pers Commun 7(5):16-27

Traffic Safety Facts 2010. Washington, DC 20590 National center for statistics and analysis

Trucks V (2015) European accident research and safety report 2MI3

Tulu GS, Washington S, Haque MM, King MJ (2015) Investigation of pedestrian crashes on two-way two-lane rural roads in Ethiopia. Accid Anal Prev 78:118-126

Tung LC, Mena J, Gerla M, Sommer C (eds) (2013) In: A cluster based architecture for intersection collision avoidance using heterogeneous networks. 12th annual mediterranean Ad Hoc Networking Workshop (MED-HOC-NET). IEEE, New York

Uselmann DJ, Uselmann LM (2004) Sonic blind spot monitoring system. Google Patents

Vogt M (ed) (2016) In: Electromobility in daily life-are you still exploring or riding already? Internationales Stuttgarter Symposium. Springer, Berlin

von Eichhorn A, Zahn P, Schramm D (2013) In: A warning algorithm for intersection collision avoidance. advanced microsystems for automotive applications. Springer, Berlin. p. 3-12

Wachtel A, Lewiston D (1994) Risk factors for bicycle-motor vehicle collisions at intersections. ITE J 64(9):30-35

Waizman G, Shoval S, Benenson I (2015) Micro-simulation model for assessing the risk of vehicle-pedestrian road accidents. J Intell Transp Syst 19(1):63-77

Wan J, Zhang D, Sun Y, Lin K, Zou C, Cai H (2014a) VCMIA: a novel architecture for integrating vehicular cyber-physical systems and mobile cloud computing. Mobile Netw Appl 19(2):153-160

Wan J, Zhang D, Zhao S, Yang L, Lloret J (2014b) Context-aware vehicular cyber-physical systems with cloud support: architecture, challenges, and solutions. IEEE Commun Mag 52(8):106-113

Wegman F (2004) In: Fewer crashes and fewer casualties by safer roads. SWOV institute for road safety research

WHO (2007) Youth and road safety

WHO (2009) Global status report on road safety: time for action. World Health Organization, Geneva

Wolf M (2014) In: High-performance embedded computing: applications in cyber-physical systems and mobile computing. Newnes

Work D, Alexandre B, Quinn J (2008) Automotive cyber physical systems in the context of human mobility. In: National workshop on high-confidence automotive cyber-physical systems, Troy, MI

Xiang X, Qin W, Xiang B (2014) Research on a DSRC-based rear-end collision warning model. IEEE Trans Intell Transp Syst 15(3):1054-1065

Zahrani MS, Ragab K, Haque AU (2011) Design of gps-based system to avoid camel-vehicle collisions: A. Asian J Appl Sci 4(4):362-377

Zhang Y, Qiu M, Tsai CW, Hassan MM, Alamri A (2015) In: Health-CPS: healthcare cyber-physical system assisted by cloud and big data

Zhao J (ed) (2011) Road traffic safety evaluation index system based on complex system-entropy theory. In: 2011 international conference on electric technology and civil engineering (ICETCE). IEEE, New York

\section{Submit your manuscript to a SpringerOpen ${ }^{\circ}$ journal and benefit from:}

- Convenient online submission

- Rigorous peer review

- Immediate publication on acceptance

- Open access: articles freely available online

- High visibility within the field

- Retaining the copyright to your article

Submit your next manuscript at $>$ springeropen.com 